\title{
Formal Analysis of the Shlaer-Mellor Method: Towards a Toolkit of Formal and Informal Requirements Specification Techniques*
}

\author{
R.J. Wieringa and G. Saake ${ }^{\mathrm{b}}$ \\ aFaculty of Mathematics and Computer Science, Free University, Amsterdam, The Netherlands; \\ bInstitut für Technische Informationssysteme, Fakultät für Informatik, Otto-von-Guericke-Universität Magdeburg, Magdeburg, Germany.
}

In this paper, we define a number of tools that we think belong to the core of any toolkit for requirements engineers. The tools are conceptual and hence, they need precise definitions that lay down as exactly as possible what their meaning and possible use is. We argue that this definition can best be achieved by a formal specification of the tool. This means that for each semi-formal requirements engineering tool we should provide a formal specification that precisely specifies its meaning. We argue that this mutually enhances the formal and semi-formal technique: it makes formal techniques more usable and, as we will argue, at the same time simplifies the diagram-based notations.

At the same time, we believe that the tools of the requirements engineer should, where possible, resemble the familiar semi-formal specification techniques used in practice today. In order to achieve this, we should search existing requirements specification techniques to look for a common kernel of familiar semi-formal techniques and try to provide a formalisation for these.

In this paper we illustrate this approach by a formal analysis of the Shlaer-Mellor method for object-oriented requirements specification. The formal specification language used in this analysis is LCM, a language based on dynamic logic, but similar results would have been achieved by means of another language. We analyse the techniques used in the information model, state model, process model and communication model of the ShlaerMellor method, identify ambiguities and redundancies, indicate how these can be eliminated and propose a formalisation of the result. We conclude with a listing of the tools extracted from the Shlaer-Mellor method that

Correspondence and offprint requests to:

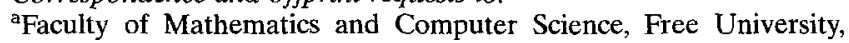
DeBoelelaan 1081a, 1011 IV, Amsterdam, The Netherlands; Email: roelw@cs.vu.nl. http://www.cs.vu.nl/ roelw we can add to a toolkit that in addition contains $L C M$ as formal specification technique.

Keywords: Requirements specification; Object-oriented analysis; Formal specification; Dynamic logic

\section{Introduction}

We view requirements engineering as the analysis of user or market needs with the aim of identifying, first, product objectives and second, externally observable product behaviour that would satisfy these objectives. We focus in this paper on techniques for the specification of required external behaviour of software products. Just as in other branches of engineering, designers need a collection of tools to specify software product requirements. A good example of such a toolkit for industrial product design (which is external design) is the collection of design methods described by Jones [1] in 1970. In a similar way, toolkits for software product specification are defined by Jensen and Tonies [2], Birrel and Ould [3] and Wieringa [4,5]. The tools that we have in mind are conceptual. They are used to reduce uncertainty about the final product by giving heuristics for making an (external) design decision and by representing the result of this decision. It is possible to supplement them with software tools such as diagram editors that take over the clerical parts of the usage of the conceptual tools. Just as in other branches of engineering, the software tools play a supporting role with respect to the usage of the conceptual tools.

The toolkit approach must be contrasted approaches in which a single method is used such as SSADM or Yourdon structured analysis. In the extreme case, 
methods like these consist of a set of manuals that prescribe a particular sequence of steps in which particular todls must be used. The advantage of a toolkit approach is that it frees the engineer from the obligation to perform tasks that are prescribed by a method but not called for by the development situation, and opens his or her mind to tools that could help to improve the quality of the final product. To get the most out of tools, our attitude towards them should be eclectic. Note that we do not advocate the abolition of method. Rather, we favour the description of the "directions for use" of requirements engineering tools in such a way that the tools can be used in different ways of working.

Requirements engineering is not yet in a state where a generally accepted set of tools has been defined. There is a variety of structured analysis methods [6-10] and an even larger variety of object-oriented methods [11-18] that jointly offer a confusing multitude of techniques and notations. It is hard to see which techniques and notations are variants of each other and which are compatible. Putting all of these together, we most certainly do not have a coherent toolkit. The addition of yet another notation to the forest of techniques would not help in clarifying the situation either. Behind the syntactic differences there is a large overlap in these methods but there are also many real differences. In order to define a usable toolkit, we have to dig behind the surface and identify a set of tools that covers the area where these methods overlap and supplement this with additional tools that are useful but do not occur in all methods. In addition, in order to increase the utility of the tools, they should resemble as much as possible the tools that software engineers are currently familiar with. This does not mean that no new tools should be defined. We think that current research in object-oriented methods has resulted in some interesting and useful additions to the more classical techniques. However, it does mean that in order to define a useful toolkit, we should not forget to include the tools that have proven their value in a number of widely used methods.

In addition to claiming that we need a set of methodindependent tools that are, where possible, familiar to the software engineer, we claim that the definition of these tools should be done by formal means even if the tools themselves are semi-formal. By ensuring that semi-formal notations such as diagrams have formal counterparts, we eliminate redundancy, inconsistency, and ambiguity in the semi-formal notations and this makes them easier to use in practice. For example, we will show in this paper that the specification of object behaviour and object communication by means of diagrams can be simplified considerably if we take care that the diagrams have precisely defined counterparts in a formal specification. This use of formal specifications does not require practitioners to ever write one letter of a formal specification, but it does demand from the tool-builders that they provide a formal theory for each tool that they produce. The situation is similar with other engineering tools: the user of a hammer does not need to have any knowledge of metallurgy or classical mechanics, but the manufacturer of a hammer does.

Formal techniques not only help in the definition of conceptual tools and techniques, they also help in the definition of software tools that support the use of conceptual tools. It is one of the hallmarks of engineering that the behaviour of a product is predicted before it is implemented; this in turn requires specification of external product behaviour before the product is implemented. The conceptual tools for requirements specification are used for precisely this: to specify external product behaviour. They also should allow prediction of product properties from the specification. But this ability requires that the semi-formal notations have a formal semantics. Once we have this formal semantics, we can build software tools that allow us to predict product properties by, for example, performing reachability analysis or by animation of possible product behaviours. Backing up semi-formal notations by formal semantics therefore helps us to improve the software product engineering process. The Statemate notation and tool is a good example of this mutual enhancement of formal techniques and semi-formal notations $[8,19,20]$.

Research in the integration of formal and semiformal techniques is scarce. Most research in this area has been done on the integration of structured analysis with formal specification techniques. France and Larrondo-Petrie [21] give a survey. In a recent paper, Bates et al. [22] present an integration of $Z$ with Fusion. They exclude life cycle specification from their formalisation. Feijs, Jonkers and Middelburg look at a large number of semi-formal diagram techniques, such as message sequence charts, dataflow diagrams and HOOD diagrams, and show how these can be combined with the wide-spectrum language COLD [23]. They do not analyse any existing semi-formal specification method in detail. Bourdeau and Cheng [24] show how an OMT object diagram can be given a formal semantics in the Larch specification language. They ignore the functional and dynamic model of OMT. In a previous paper, we analysed all three models of OMT [18] using the formal specification language TROLL [25]. One of us (Wieringa) analysed entity-relationship modelling, structured analysis and JSD to define an initial version of a toolkit for conceptual modelling of software 
product requirements $[4,26]$. In this paper, we analyse the Shlaer-Mellor method for object-oriented requirements specification $[16,17]$, called object-oriented analysis (OOA) henceforth. Like OMT, OOA is well known and is described in publicly available literature. It is additionally important because it can be viewed as a further evolution of classical structured analysis for real-time systems [10] and contains notations and ideas that are familiar to users of structured analysis. OOA is therefore a good place to look for notations and techniques that are familiar to software engineers and that are useful additions to our toolkit.

The structure of this paper is as follows. In Section 2, we give an overview of the deliverables of OOA. In Sections 3-6, we analyse the major deliverables of the method, the information model, the state model, the process model and the object communication model of a system. We identify the notations used, analyse these from a methodological point of view, and indicate whether and how the notations can be formalised. As the vehicle for formalisation we use a syntactically sugared version of dynamic logic called LCM (Language for Conceptual Modeling). The focus remains on the formal analysis of OOA, however. The paper is not meant to be an introduction to LCM. Section 7 concludes the paper with a summary, discussion of results and a list of some topics for further research.

\section{The Deliverables of the Shlaer-Mellor Method}

The Shlaer-Mellor method divides the system under development into domains that each pertains to one subject matter. Each domain is inhabited by its own set of objects that behave according to a closely related set of rules and policies. For example, two domains of a railroad control system would be railroad operations and user interfaces. Domains are further divided into subsystems such that each object exists in exactly one subsystem and never moves between subsystems. Example subsystems in the domain of railroad operations are train dispatch and track management. Each subsystem is a collection of interacting objects. For each subsystem, the following models are produced.

1. The information model shows the classes of objects that make up the subsystem, and the relationships between these object classes. The information model uses an extension of the ER notation.

2. A state model represents the behaviour of all instances of a class. State models are only specified for classes whose instances have interesting behav- iour. The state model of a class contains state transition diagrams, possibly supported by state transition tables and an event list.

3. The process model represents the processing done when the system receives a stimulus. The core of the process model is an action data flow diagram (ADFD) that is specified for each action in each state model and which shows which processes are performed during the action. Each of these processes must be documented by a process specification. The process model may be supplemented by a listing of all processes and the place where they are used and by an object access model (OAM), which lists all synchronous inter-object accesses that occur during any action.

4. The object communication diagram represents asynchronous messages sent among objects by means of a directed graph.

We restrict ourselves to a formal analysis of the subsystem model. Whenever we speak of "the system" below we mean the subsystem that is modelled.

\section{The Information Model}

\subsection{Structure}

The information model in OOA uses a variant of the Entity-relationship (ER) notation. Figure 1 gives an example of the notation used in the information model.

- Each box in the diagram represents an object class. In OOA this is called an object and instances are called object occurrences. In order to reduce the gap with other methods and prepare for the addition of this notation to our toolbox, we talk of object classes and call occurrences objects.

- Each object class has an identifier, defined in OOA as a collection of one or more attributes whose combination always has a unique value for each instance of the class. These attributes are marked by an asterisk.

- All relationships in the information model are binary. They are represented by bidirectional arrows. Cardinalities are one-one, many-one, or many-many, with mandatory or conditional participation on either or on both sides. A single arrowhead on one side of an arrow means exactly 1 if that side has mandatory participation, and it means 0 or 1 if that side has conditional participation. A double arrowhead on one side of an arrow means $\geq 1$ if that side has 


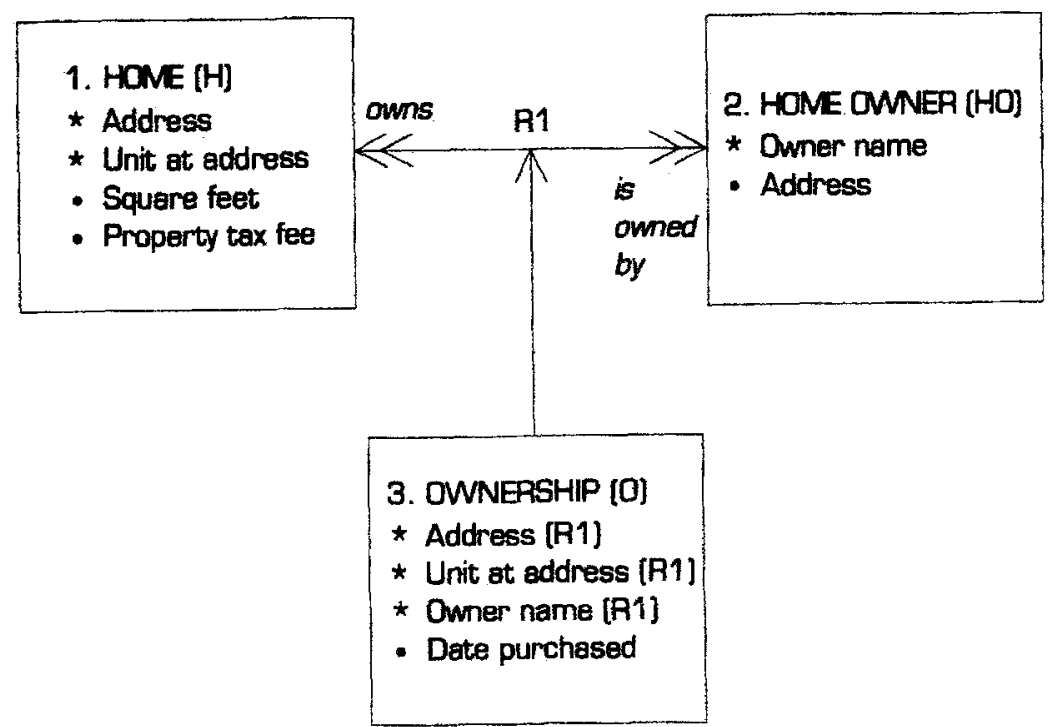

Fig 1. An associative object class. [Object Lifecycles: Modeling the World in States by Schlaer \& Melior (c) 1991. Reprinted by permission of Prentice-Hall Inc., Upper Saddle River, NJ.]

mandatory participation and it means $\geq 0$ if it has conditional participation. Default participation is mandatory. Conditional participation is represented by the letter "c" at the arrowhead.

OOA allows the definition of relationship attributes. As shown in Fig. 1, these are collected into something called an associative object class (OWNERSHIP), which is connected by an arrow to the relationship whose attributes are represented.

Subclasses and superclasses are represented as shown in Fig. 2. The intention is that in each state of the system, the set of existing instances of the superclass is partitioned by the sets of existing instances of the subclasses. Each existing instance of TANK is therefore also an existing instance of exactly one of the TANK subclasses. OOA allows class migration. For example, if
MIXING_TANK would be partitioned into subclasses $U N A S S I G N E D$ and $A S S I G N E D$, we can allow a mixing tank to migrate between these two subclasses.

\subsection{Methodological Analysis}

The information model embodies fairly standard and accepted ideas, so it is certainly a candidate for addition to our toolkit. The roots of information modelling lie in ER modelling [27] and semantic modelling [28,29]. Closely related approaches with different notational conventions can be found in other object-oriented methods, such as OMT [18] and the Coad-Yourdon method [12]. To prepare for formalisation, we analyse two important aspects of information modelling, object identification and the taxonomic hierarchy.

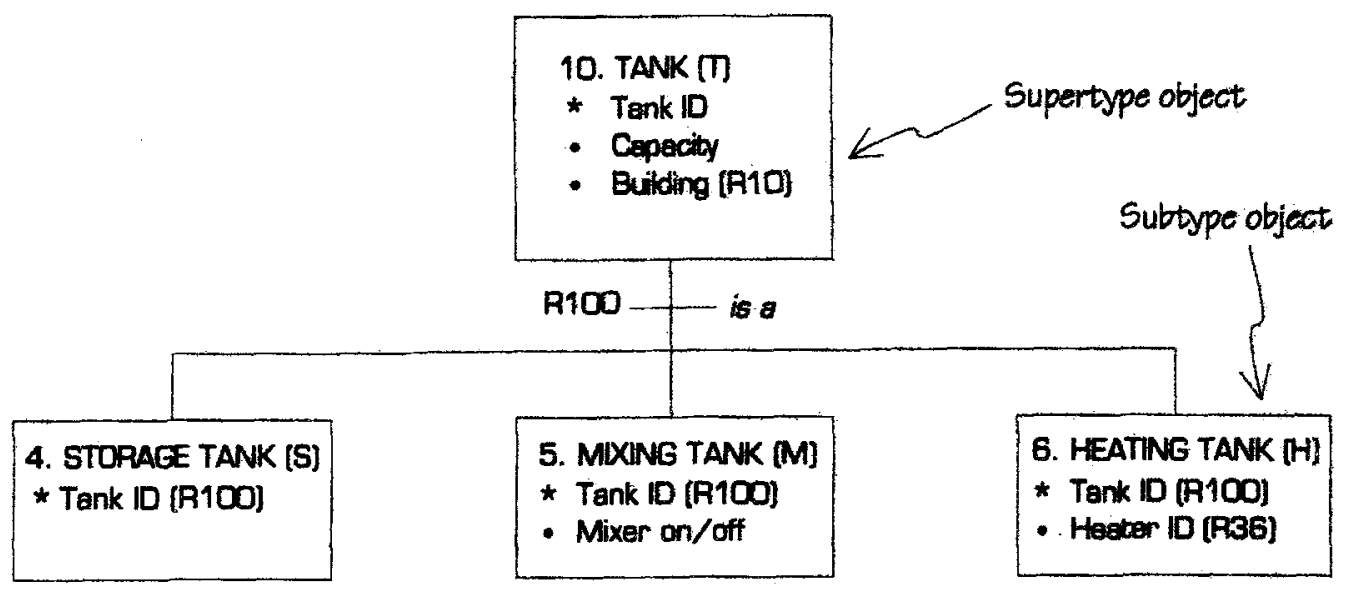

Fig 2. Representation of specialisation. [Object Lifecycles: Modeling the World in States by Schlaer \& Melior (C) 1991. Reprinted by permission of Prentice-Hall Inc., Upper Saddle River, NJ.] 
The importance of object identification goes beyond object-oriented requirements specification..Any administration that wants to keep track of objects in the real world must devise a way to identify these objects [ 30 , 31]. The only way to do this is to assign a proper name to each relevant object such that there is a one-one relationship between these names and the identified objects that, once established, never changes. Thus, the set of links between proper names and identified objects grows monotonically. These proper names can then be used as object identifiers in the system. In order to achieve thiis, the name must not designate any property of the object that can change. In practice, this means that the name does not carry information about the state of the object and it is indivisible, that is, it cannot be decomposed into meaningful parts. We therefore deviate from the OOA way of identifying objects and require object identifiers to be atomic.

OOA rightly allows objects to migrate between classes. This has an important methodological consequence that is, however, not remarked by Shlaer and Mellor, namely that in object migration an instance may become a member of a subclass without creating an instance of a superclass [32]. For example, if an instance of MIXING_TANK migrates from UNASSIGNED to ASSIGNED, then the set of existing instances of UNASSIGNED is decreased by one without a change in the set of existing instances of $M I X I N G \_T A N K$. Contrast this with the non-migratory case: If the partitioning of TANK into STORAGE TANK, MIXING_TANK and HEATING_TANK is static, that is migration between these subclasses is not allowed, then a change in the existence set of $M I X$ $I N G_{-} T A N K$ implies a change in the existence set of TANK. In practice, it is very difficult to find a taxonomic partition of a class that does not allow migration. For example, it is not inconceivable that the three subclasses of TANK indicate three ways of using a tank, and in that case a tank could migrate between these subclasses.

Migratory and non-migratory subclassing share the property that an instance of a subclass is also an instance of the superclass. This is different in the case of role-playing, treated next. Is an EMPLOYEE instance identical to a PERSON instance? At first sight, the answer seems obviously positive, until one asks whether one person can be several employees (at the same time or in series). If someone has two jobs, each with its own employee number, is this person one or two employees? If we want to keep track of the number of jobs a person has, then it may be preferable to model this person as playing two employee roles. Correspondingly, this person will have two employee identifiers and then an employee is not identical to a person. Nevertheless, there is a close relationship between employees and persons, because each employee can only exist as a role of a person. Roles can be modelled by means of a many-one relationship from EMPLOYEE to PER$S O N$. Summing up, the is_a arrow in an information model may represent two different situations (migratory and non-migratory subclassing) and in some cases, specialisation is more suitably modelled as role-playing, which can be represented by a many-one relationship. Any formalisation will have to take these different cases of specialisation into account.

\subsection{Formalisation}

In this subsection, we briefly sketch a formalisation of the information model. This formalisation has been described in more detail elsewhere $[33,34]$ but we need it here in order to understand the formalisations of the other parts of OOA specifications.

The information model leaves open what the type of attributes is but in a formal specification that is counterpart of the information model, this type must be explicitly specified. We therefore assume that there is a mechanism to specify abstract data types (ADTs) and that a number of standard ADTs are available to the formal specification, such as NATURAL, STRING and $M O N E Y$. In this paper, we assume order-sorted equational logic with initial algebra semantics as the mechanism to specify ADTs. We refer to the literature for the formal details [35-37] and here give an informal indication of what this means.

- An ADT is a set, called a sort, with a number of operations.

- ADTs are partially ordered to represent the subtype relation on ADTs. The subtype relation implies a subset relation on sorts. For example, in the partial ordering of number types NATURAL $\leq$ INTEGER represents the fact that NATURAL is a subtype of INTEGER; this implies that the sort of naturals is a subset of the sort of integers.

- The properties of operations are given by means of equations. For example, a property of addition on the sort NATURAL is that $\operatorname{succ}(n+m)=\operatorname{succ}(n)+m$, where succ is the successor function.

- Further properties can be derived using equational logic, which is based on the substitution of equals for equals.

- Each closed term (a term not containing variables) is the syntactic representation of a value. In the initial algebra semantics, all values are named by a closed term and different closed terms represent the same value if and only if they can be proved equal by using 
the axioms in the specification and the rules of equational logic.

We define the extension of an ADT as the set of all instances of the type in the intended semantics (in our case, in the initial algebra semantics); this is the set of all values that can be denoted by a closed term.

Next, we need to formalise the concept of class. The idea is simply to formalise each attribute as a function that accepts an object identifier as argument and delivers an attribute value as result. The methodological consequence of this idea is that identifiers are themselves not attributes and that objects can never change identifiers. This agrees with our methodological analysis.

In the formal specification, we now require for each object class that a corresponding identifier type has been specified, contains all identifiers that will ever be needed for objects of that class. The identifier type has the same name as the class. Each attribute is now formally declared to be a function on the identifier type of the class. For example, if we assume in Fig. 1 the name of a customer is a string, owner_name is a function $H O M E \_O W N E R \rightarrow S T R I N G$.

In any state of the system only some identifiers will identify existing objects. To single out those identifiers, we assume a predicate Exists that is applicable to all identifiers and that only evaluates to true for identifiers of existing objects. The extension of the Exists predicate is the set of identifiers for which it evaluates to true. Exists may have different extensions in different states. The existence set of a state is the set of all identifiers for which Exists evaluates to true. If Exists $(n)$ is false for an identifier $n$ then $n$ may have existed in the past or it may come into existence in some possible future. If it has existed in the past, it is not available for use as identifier anymore. This is formalised by maintaining a unary predicate $U$ sed that is true for all identifiers that have ever been added to the existence set. The extension of this predicate is nondecreasing.

Figure 3 shows an example of a formalisation of part of the information model in Fig. 1 in LCM. The specification of object class $H O M E$ is syntactic sugar for the declaration of an identifier type $H O M E$ and of functions address : HOME $\rightarrow$ STRING etc. The set of attributes \{address, unit_at_address\} is declared to be a key. This is syntactic sugar for an axiom which says that if two existing homes have the same value for this set of attributes, then they are equal:

$$
\begin{aligned}
\operatorname{address}(h 1) & =\text { address }(h 2) \text { and unit_at_address }(h 1) \\
= & \text { unit_at_address }(h 2) \rightarrow h 1=h 2
\end{aligned}
$$

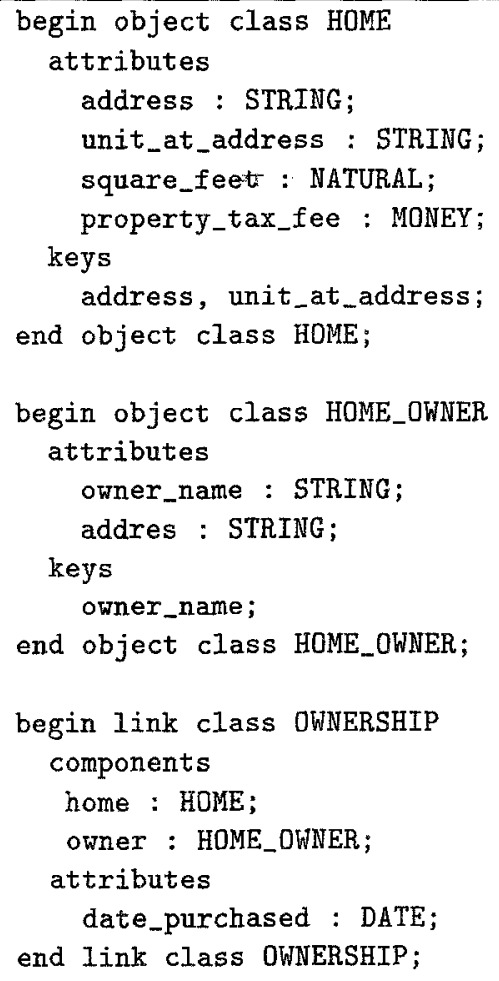

Fig. 3. Specification of a relationship with attributes in LCM.

where $\rightarrow$ stands for logical implication. Note that we formalize the identifier concept of OOA as keys in LCM.

A relationship instance is a labelled tuple of component objects called a link in LCM; a relationship is called a link class in LCM. The specification of a link class must declare the components of the links. The components section of the specification of OWNERSHIP is syntactic sugar for the specification of an identifier type whose values have the following structure:

< home: h, owner: o >

where $h$ and $o$ are identifiers of type HOME and HOME_OWNER, respectively. The labels home and owner in the link are the names of projection functions that retrieve the components of these identifiers. These projection functions are called component functions in LCM. Thus, we have a function

home: OWNERSHIP $\rightarrow$ HOME

defined by

home (<home: $h$, owner: $o>)=h$

The components section generates implicit component existence axioms such as the following: 
forall os : OWNERSHIP :: Exists(os) $\rightarrow$ Exists(home(os))

Cardinality constraints are formalised by placing constraints on the component functions of a link class. For example, if each existing $H O M E$ must participate in an existing OWNERSHIP link, then the home component function is declared to be a surjection and if each existing $H O M E$ must participate in at most one link, then the component function home is declared to be an injection. The axioms for this are straightforward and are omitted here.

Attributes applicable to relationships are formalized as functions that take link identifiers as arguments. For example, the date attribute of an OWNERSHIP instance is a function OWNERSHIP $\rightarrow$ DATE. Like all attributes, this attribute can be updated. By contrast, component functions cannot be updated. If we replace the component $h$ in the link identifier <home:h, owner:o $>$ by a different identifier $h^{\prime}$ then the result is an identifier of a different link, not an updated link identifier. Apart from identification, LCM treats link classes in all respects in the same way as object classes.

Before we turn to the formalisation of taxonomic structures, we briefly sketch the intended semantics of the specification. Formal details are given elsewhere $[34,38]$.

- The specification is interpreted in a state transition system (also called a Kripke structure).

- All states in the state space of this system have the same domain, which is the union of the ADTs presupposed by the specification. This means that all constants and operations on ADTs have the same interpretation in all states of the system; for example, it is impossible that after a state change, the meaning of integer addition would be changed. It also means that all object identifiers, which are instances of identifier types, are available in all states of the system.

- The extension of the existence predicate may vary in different states of the system. In each state, the predicate will evaluate to true for some identifiers. Whenever an identifier is added to the extension of Exists, it is also added to the extension of Used. An identifier can only be added to the extension of Exists if it is not already in the extension of Used. Once it is in the extension of $U$ sed it is never removed from this extension.

- Attributes are interpreted as updatable functions. In different states of the system, one attribute can be interpreted as different functions.
Finally, we sketch a formalisation of subclassing. This formalisation is discussed in detail by Wieringa et al. [32]. If objects cannot migrate between subclasses of a certain class $C$, then the specialisation of $C$ can be represented by a partial ordering of identifier types. For example, if Fig. 2 represents a static partitioning of TANK, then the identifier type of STORAGE_TANK is a subtype of the identifier type of TANK. This means that the sort of all TANK identifiers is partitioned by the sort of identifiers of STORAGE_TANK, MIXING_ TANK and HEATING_TANK. Because identifier types are ADTs and we use ADTs as fixed domain of all possible states of the system, this partitioning is the same in all possible states of the system, so that an identifier never changes its type; this agrees with our methodological analysis.

If migration between subclasses is to remain possible, then specialisation must be formalised by means of a class predicate that can change its extension. For example, if MIXING_TANK is partitioned into subclasses UNASSIGNED and ASSIGNED with the intention that mixing tanks can migrate between these two subclasses, then two predicates Unassigned and Assigned are defined, whose argument is of type MIXING_TANK. A mixing tank would then migrate between its subclasses by changing the truth value of these class predicates.

We noted earlier that role-playing can be represented by a many-one relationship with the constraint that an existing role can only be played by an existing player. This poses no special problems for the formalisation.

\section{The State Model}

\subsection{Structure}

For each object class or relationship with interesting behaviour, a state model can be defined that represents the typical life cycle of class instances. The instantiation of a state model for class instances is called a state machine in OOA. Each class instance has a state attribute that indicates the current state of the object in its state machine. State models and state machines are finite state automata, which can be represented in two (equivalent) ways: the Mealy and the Moore representation, shown in Fig. 4. Both representations in the figure show a transition triggered by input event $e$. The transition in turn triggers output action $a$. In the Mealy representation $a$ is performed during the transition and in the Moore representation, $a$ is performed upon arrival in the destination state. Hence, in the Mealy 


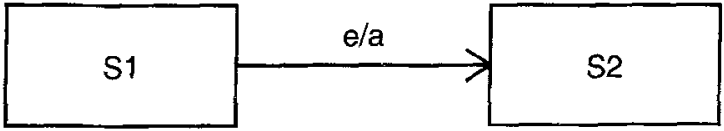

Mealy representation

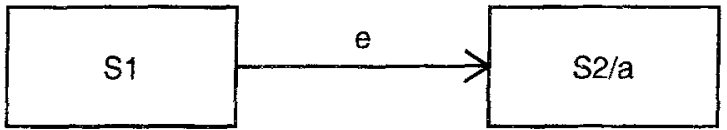

Moore representation

Fig. 4. The Mealy and Moore representations of finite state machines.

representation, output actions are attached to transitions and in the Moore representation, output actions are attached to states. A Moore representation can be transformed into an equivalent Mealy representation by moving the output action of each state to the incoming transitions of the state; conversely, a Mealy representation can be transformed into a Moore representation by introducing additional states in such a way that the incoming transitions of each state generate the same output actions, and then moving the output actions to the states $[39,44]$. The Moore representation is convenient if all transitions entering a state generate the same actions; otherwise, the Moore representation tends to have more states than the Mealy representation. This is illustrated by the methodological analysis below.

OOA uses the Moore representation of finite state machines. An example of a state model using the Moore representation is given in Fig. 5. Each event in a state model is an incident to which an object in the system must respond. The event may be generated by an object in the system, or it may be generated in the environment of the system. The state machine responds to an event by performing an action. OOA allows the following actions $[17,45]$ :

- Read or write attributes of any object in the system.

- Perform any calculation.

- Generate an event. This event may be received by the environment of the system, by another object, or by the object itself (in its next transition).

- Create, delete, set, reset or read a timer. A timer is an object that functions as an alarm clock. Any number of timers may be created in the system.

Relationships may have two state models, one that describes the life cycles of its instances and one, called the assigner, that monitors the creation of relationship instances. There is at most one assigner for each relationship. We ignore assigners in what follows, because they can be represented in the same way as state models that describe the life of instances.

\subsection{Methodological Analysis}

We will use the account state model of Fig. 5 as a vehicle to argue that an equivalent Mealy representation of the state model is considerably simpler and can be defined in such a way that it contains less implementation bias. To argue this, we start by noticing that there are two kinds of states in the account state model, stable and transitory. In a stable state of an object, the object is ready to respond to events initiated by its environment, and in a transitory state the object is busy responding to an event that it has received. When the object is busy responding to an event, it will queue incoming events so that it can respond to them later. The concept of transitory state is well known from the Ward-Mellor method of structured analysis [10]. For example, opening an account brings one into state 1 , in which the Moore automaton creates and initialises an account and generates a deposit action. The deposit action is received by the very same object and causes transition into state 2 , where the deposit is added to the balance. If the balance is non-negative, the action account_OK is generated, which causes the account to move to state 3 and if the balance is negative, the action overdrawn is generated, which causes the account to move to state 4 . The account then settles in state 3 or 4 , that is, these are stable states in which the object can respond to events that it receives from the environment. The states that the account meets on the way are transitory. In fact, states 3 and 4 are the only stable states in the diagram.

Transitory states can be used to factor out common computations among different transitions. For example, state 2 factors out the action of depositing money, which occurs in the initialisation transition of an account, in the deposit transition from the overdrawn state and in the deposit transition from the in good standing state. Transitory states are also useful to test a guard to determine which transition can be taken. For example, state 6 tests whether handling a check would cause the account to be overdrawn and so tests whether to go tø state 7 or state 3 .

Let us define an object transaction as an atomic interaction between the object and its environment, 
where an interaction is defined to be atomic äf its intermediary states are not observable to the environment of the object. Viewed from the environment of the object, the object is in a state where either the transaction has occurred or it has not occurred. This is the classical transaction concept known from database

\section{Account}

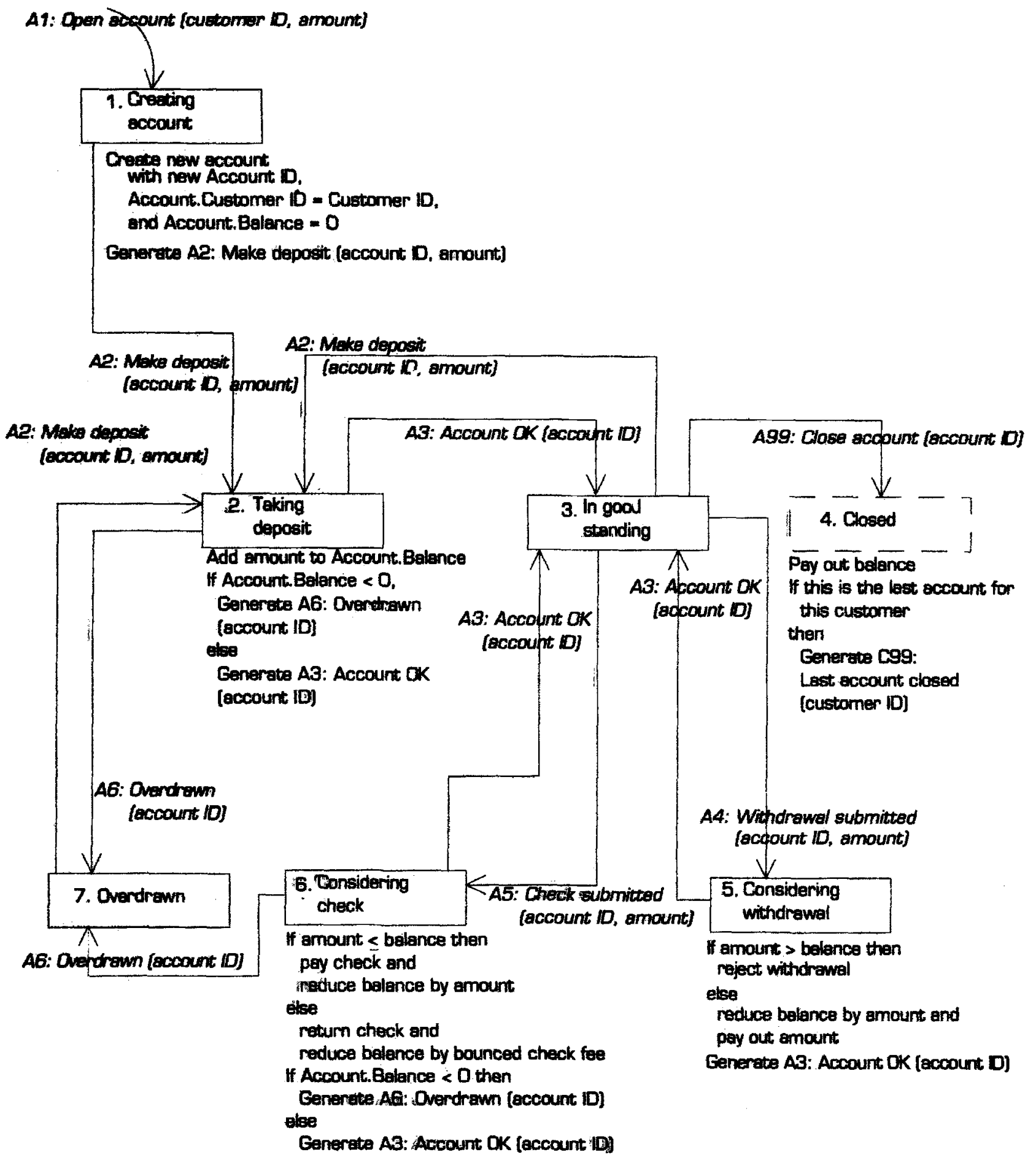

Fig 5. State models in OOA use the Moore convention. [OObject Lifecycles: Modeling the World in States by Schlaer \& Melior 11991. Reprinted by permissiom of Prentice-Hall Inc., Upper Saddle River, NJ.] 
theory [40]. An object transaction is always a sequence of one or more transitions from a stable state to a stable state, moving through zero or more intermediary transitory states that are not observable from the environment. State models with the same observable behaviour but with different sequences of transitory' states are indistinguishable for the environment and hence observationally equivalent. A representation of state models in which only stable states appear shows only essential object behaviour in the sense that it is free from any implementation bias [41]. Such a representation is therefore to be preferred to representations that are observationally equivalent but use different transitory states.

To eliminate transitory states from a state model, we must gather all actions performed during an object transaction and compress them into one action. If the object passes through a transitory state in order to decide what the next stable state is going to be, then the actions performed in this transitory state perform a test. In order to eliminate such a state, we must extend the labels of the arcs with guards which state the test in declarative form. This will become clear when we give an example in a moment.

First, in order to be able to reduce the number of states of the automaton, we move from the Moore to the Mealy representation. For example, Fig. 6 shows the Mealy automaton for handling a check obtained when we move the action of state 6 to the incoming arc. Next, we eliminate the transitory state (number 6). Because this state performs a test to determine if the next stable state is state 3 or state 7, we define guards which specify the condition under which the next state is Overdrawn or In good standing. Figure 7 shows the result. The guards are added between square brackets after the event. Note that the declarative specification of the guards is less implementation-oriented than the operational specification of the test in Fig. 6. The Mealy model of Fig. 7 merely says which events are received, which actions are generated, and which guards must be tested by the object.

Figure 8 sfrows the result of transforming the account state model by moving the actions to the incoming arcs, removing transitory states and specifying guards declaratively. (The submit check action in state overdrawn is not present in Fig. 5. We added it because there seems to be no reason to exclude it.) To summarize, the resulting Mealyy automaton illustrates three points made in the above discussion:

- Mealy automata are usually simpler than their equivalent Moore automatons because they have less states and transitions.

- Because guards are specified declaratively rather than operationally, they avoid implementation bias.

- By the elimination of transitory states, an automaton represents essential behaviour only.

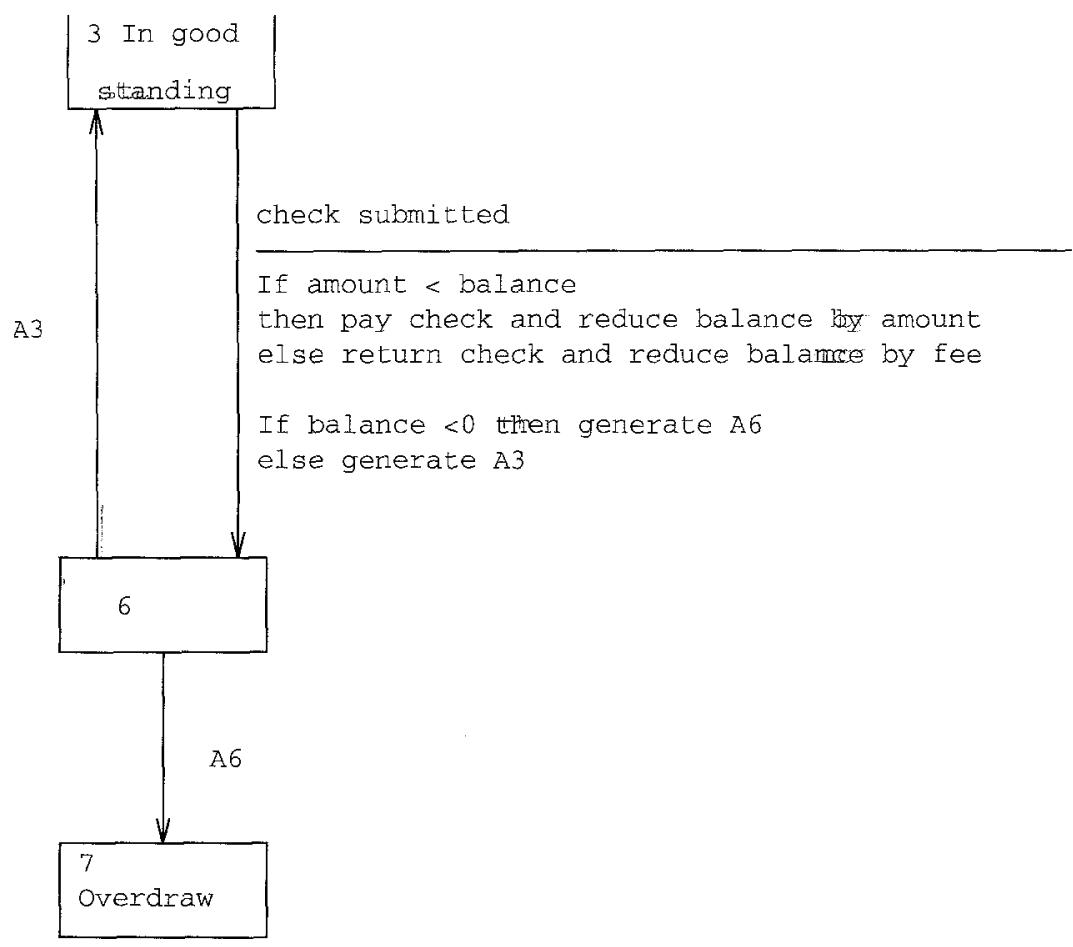

Fig. 6. Mealy automaton for harndling a check. 
submit_check(m) [balance-m $<0$ and balance-fees=0]

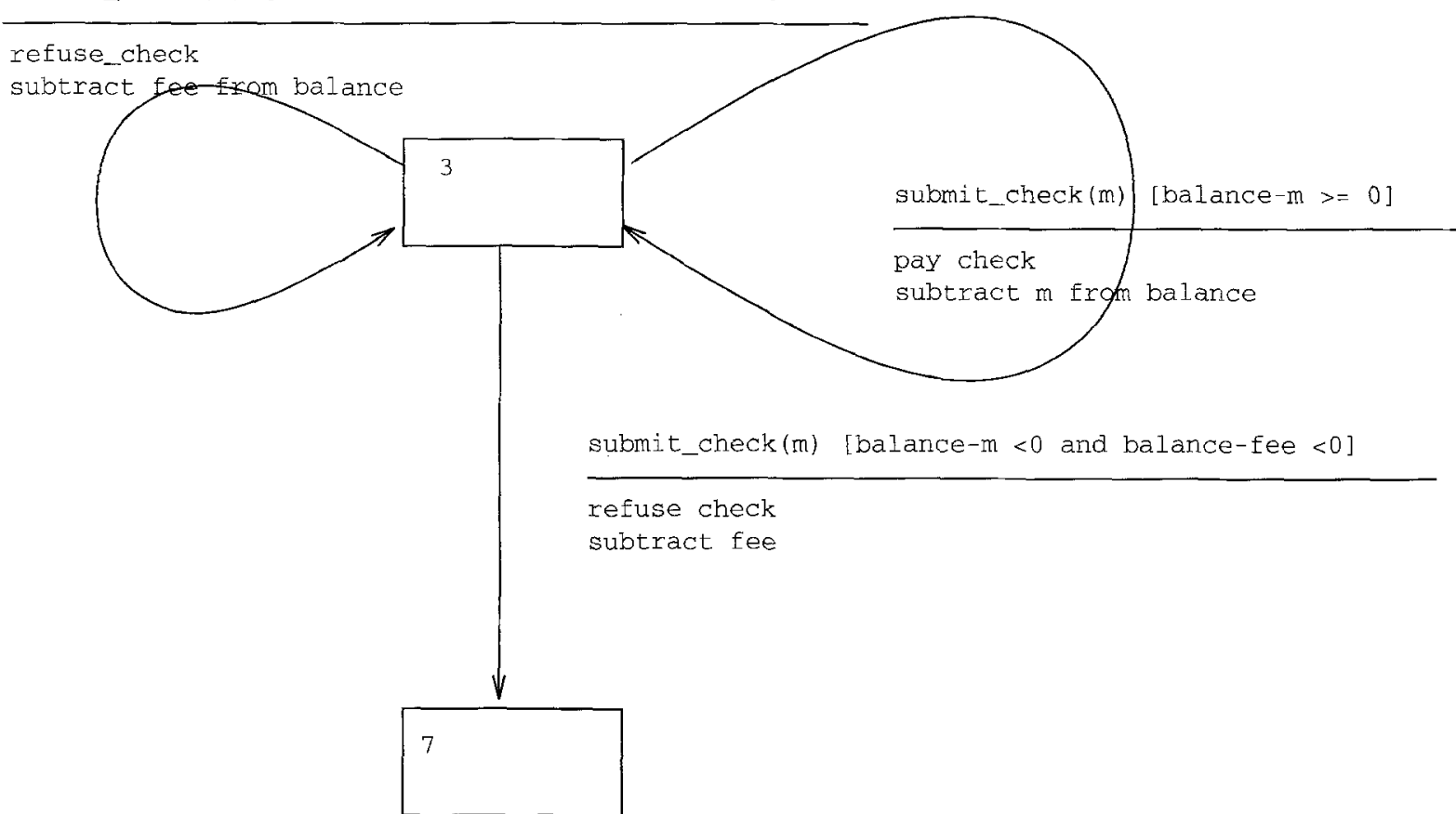

Fig. 7. Mealy automaton with declarative guard specification.

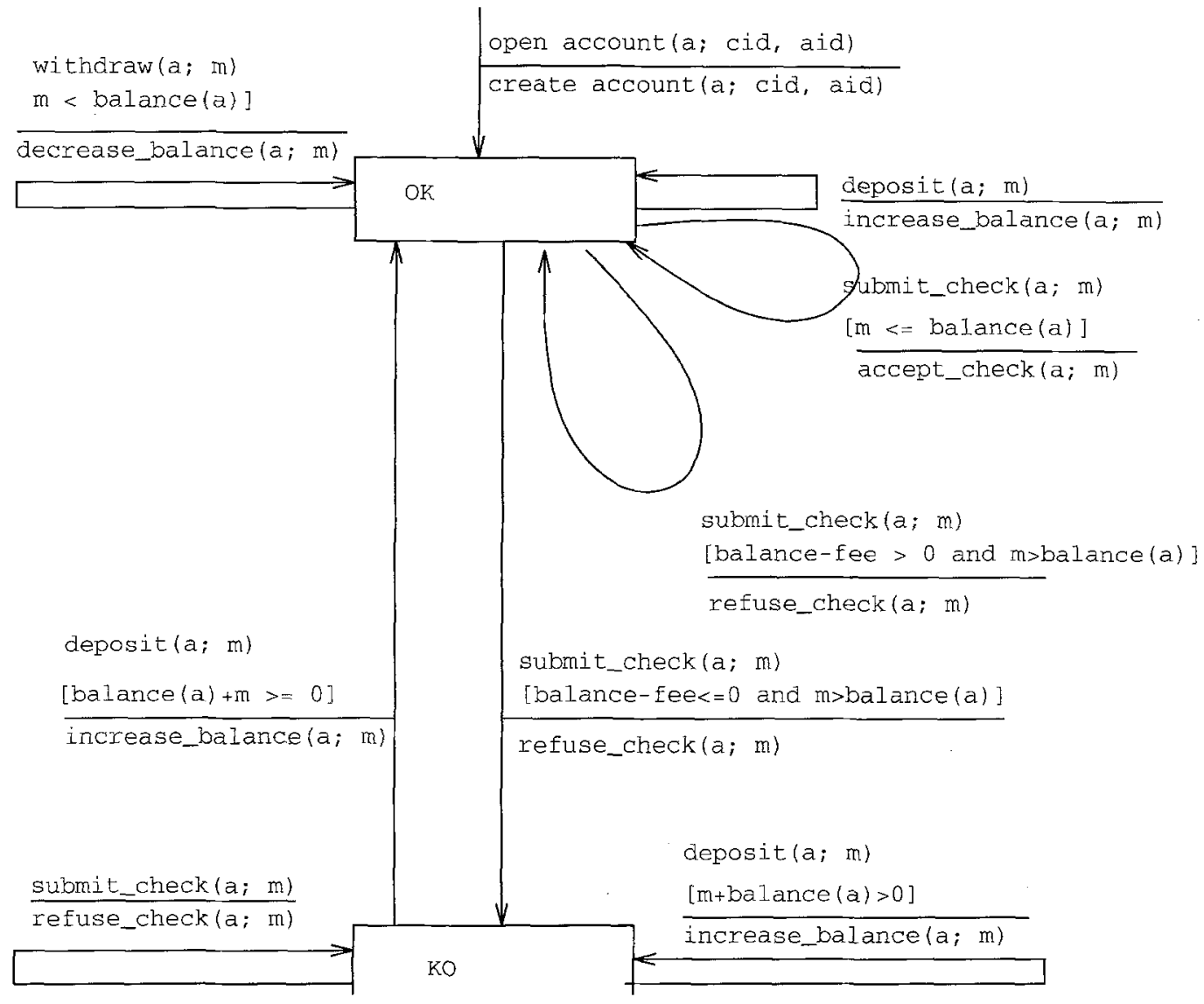

Fig. 8. Mealy automaton with guards instead of transitory states. 
We conclude that the representation of state models without transitory states and with declarative guards is to be preferred above a representation with transitory states and guards that are specified operationally. We have no preference for the Mealy or Moore notation; indeed, in general both conventions may be mixed as in the statechart convention $[8,42]$. The Moore convention should be used for states for which all incoming arcs of a state generate the same action.

\subsection{Formalisation}

When an object transaction occurs, a transition in the state machine of the object is taken. The following things happen in this transition:

- An event that triggers the transaction is received from the environment of the object.

- The guard of the transaction is evaluated.

- The action associated with the transaction may cause changes in the system (OOA allows an action to have effects anywhere).

- The action is sent to the environment of the object.

The first and last items are part of the communication structure of the system and will be specified formally when we discuss the object communication model. The second and third items concern the meaning of actions (including their guards). The meaning of actions is specified in the OOA process model and we will turn to the formalisation of this part of a transition after we analyse the process model below.

\section{The Process Model}

\subsection{Structure}

In the process model, each action is specified by an action data flow diagram (ADFD) that represents the processing performed during the action. To illustrate this, we give an ADFD for the Created state of the temperature ramp state model in Fig. 9. The temperature ramp is part of a model of a controller of a juice plant that mixes juice in a cooking tank and puts it into cans. The entire model specifies the controller for the cooking tank and the canning operation and is specified more fully in Shlaer and Mellor [43]. The temperature ramp state model of Fig. 9 contains the following actions:

- Created. Upon creation of a temperature ramp, a timer is created for this ramp, the current time and the current cooking tank temperature are stored in the attributes of the temperature ramp, and the ramp sends an event to itself to transition to the controlling state.

- Controlling. Upon entry in the controlling state, and henceforth every time the timer expires, the ramp compares the actual cooking tank temperature with the desired temperature and turns the heater on or off accordingly. If the temperature is not high enough, the time is set to 10 seconds. If the temperature is high enough, the ramp sends an event to itself to transition to the complete state.

- Complete. Upon entry in this state, the ramp sends an event to its environment that it is complete, turns off the heater, deletes the timer and deletes itself.

Figure 10 contains an ADFD for the actions performed in the Created state. An ADFD is a collection of processes (represented by ovals) and data stores (represented by two parallel lines) connected by data flows. The processes represent operations performed during the action by objects in the system. For each process in an ADFD, a process description must be produced (not shown here). The data stores in an ADFD contain the states of existing objects of a class, called a class data store, or the states of timer objects, or the current time. There may be data stores of several different classes in one ADFD. There may be at most one data store that contains the current time. Data flows represent the flow of information in the action and are represented by arrows.

Processes in one ADFD may be performed by different objects in the system. The allocation of a process to an object class is expressed by the process identifier. In Fig. 10, the following identifiers have been chosen: TR for Temperature Ramp, CT for Cooking Tank, and B for Batch. If the ADFD of an action in the state model of one class accesses the object data store of another class then this represents a synchronous communication between objects of these two classes. These synchronous communications can be represented by an object access model (OAM). The OAM is a directed graph in which the nodes represent objects and the arcs processes that perform inter-object access.

\subsection{Methodological Analysis}

There are some problems with the use of data flow diagrams in combination with object-oriented modelling techniques. First of all, data flow diagrams represent what we call the Von Neumann partitioning principle in which data is separated from processing. This partitioning goes against the object-oriented partitioning principle that data and processing should be 
grouped together $[43,45]$. Second, all the data in a data store is globally accessible to any process that accesses it [46]. This again contrasts with the object-orientedencapsulation of data with the processes that access it. Third, data flow diagrams specify the operations that take place during an action, and this tends to have a bias towards implementation [43,47]. To be completely free from any implementation bias, a declarative specification of the meaning of actions is to be preferred.

These problems with DFDs do not imply that they should be abolished altogether. Using ideas from McMenamin and Palmer [41], we can define an essential data flow model of object transactions as a data flow model with the following contents (Fig. 11):

- The state of the object is represented. by a data store.

- Each object transaction is representediby exactly one data transformation.

- Each data transformation represents exactly one object transaction.

Thus, essential data transformations have no memory. We add to this McMenamin and Palmer's idea of perfect technology, which says that an essential data flow model describes a machine with infinite processiing

TA90: Do temperature ramp [batch D. end time, end temperature]

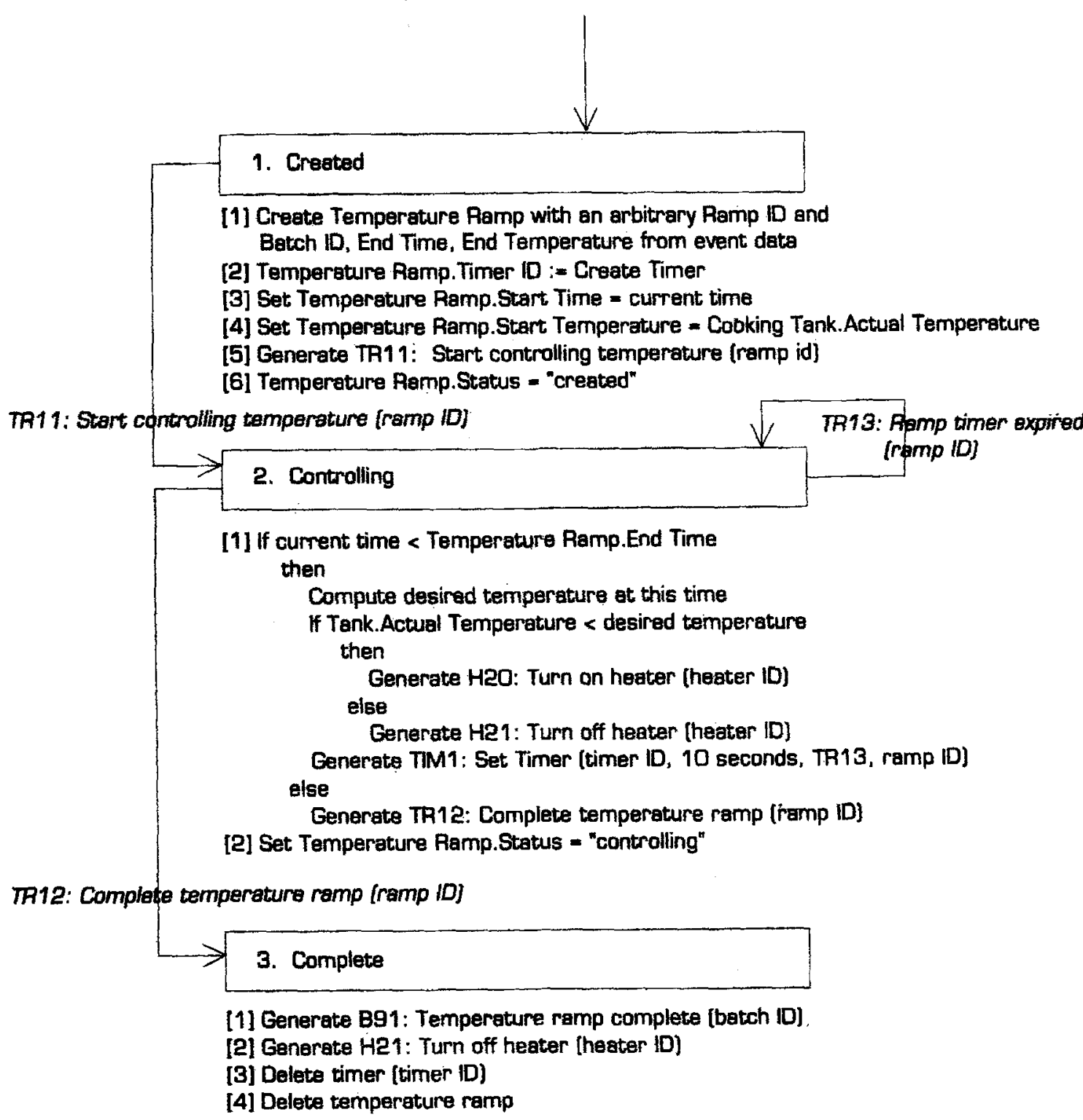

Fig 9. State model of a temperature ramp object class. [Object Lifecycles: Modeling the Worid in States by Schlaer \& Meliór@1991.

Reprinted by permission of Prentice-Hall Inc., Upper Saddle River, NJ.] 
power and unrestricted memory. This ties in with our earlier analysis of object transactions, which are atomic and'thus conceptually take no time. It implies that there are no direct data flows between the data transformations in an essential data flow model; if there were, then the connected transformations would always be executed simultaneously (because they take no time) and hence they would represent one object transaction; but this should be represented by a single data transformation. Thus, in an essential data flow model, data transformations interface only with data stores and external entities.

Figure 11 shows part of an essential data flow model for the account object class, together with a declarative specification of the two object transactions represented by the model. Inputs from the environment of the object to a transaction are represented by data flows from an external entity to the data transformation that represents the transaction, labelled by the transaction parameters that are supplied by the external entity. Values computed by the transaction should occur as transaction parameters and are labels of output data flows to external entities. Evaluation of the guard requires data from data stores. This may involve reading from any class data store. These data flows are labelled by the attributes read from the data store. Realising the effect of the transaction involves writing to the data store of the object class to which the transaction belongs. These data flows are labelled by the attributes written to the data store. This use of data flow diagrams is in the same spirit as the functional model of the new version of OMT [48].

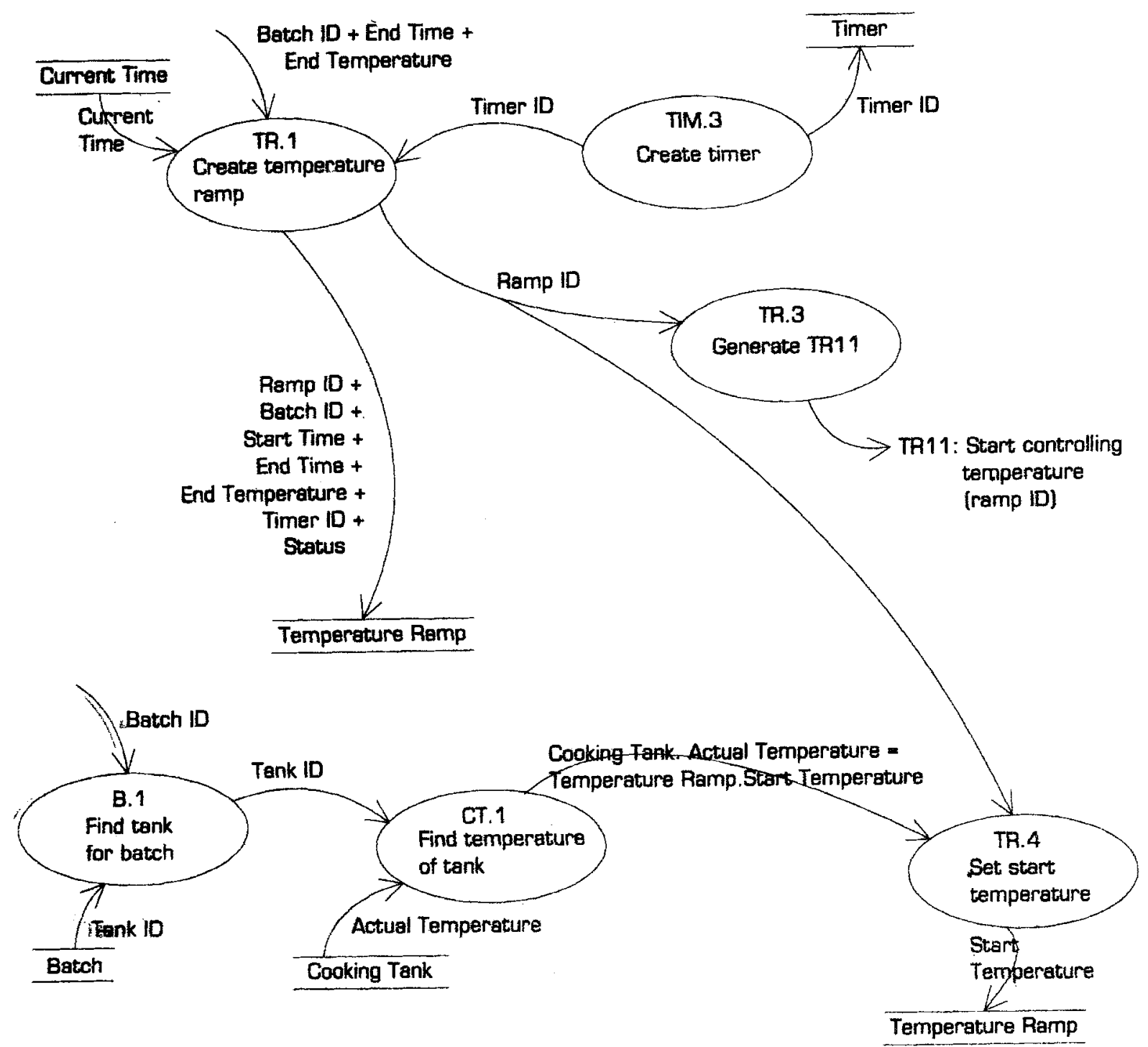

Fig 10. An action data flow diagram (ADFD) for the created state of the temperature ramp. [Object Lifecycles: Modeling the World in States by Schlaer \& Melior @ 1991. Reprinted by permission of Prentice-Hall Inc., Upper Saddle River, NJ.] 


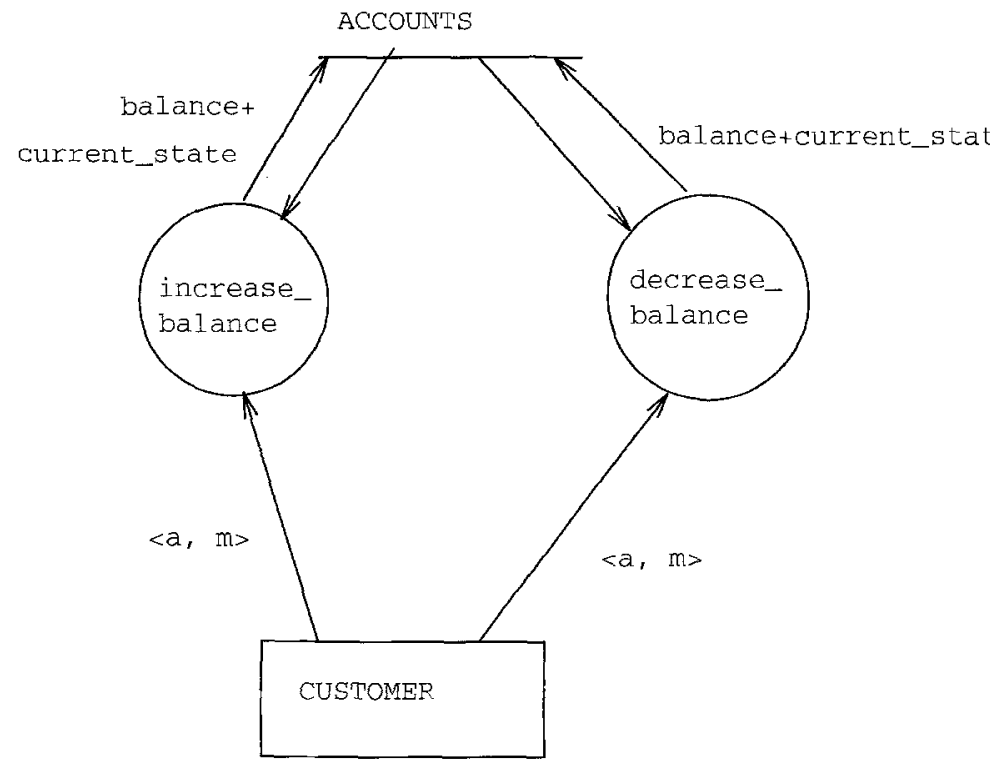

increase_balance $(a ; m)$ increases the balance of a by $m$ and sets the current state to IN_GOOD_STANDING if the result is non-negative.

decrease_balance $(a ; m)$ decreases the balance of a by $m$ only if the resulting balance would be non-negative.

Fig. 11. Data flow model for two account object transactions, with declarative (informal) transaction specifications.

There is a difference between OOA and other object oriented methods concerning the locality of action effects. OOA allows an action to change attribute values of any object in the system. This causes a number of problems. First, it is at odds with the object-oriented principle of encapsulation. Second, we will see below that communication is almost trivial to formalize if we require actions to have local effects only. For these reasons, we will add the restriction that an action should have local effects only. This restriction is visible in the essential data flow model of an object because the transformations in this model (which correspond to transitions in the state model) have write access only to the class data store of the object. The descriptions of the transformations should make clear that each transformation can only update the state of the object in whose life it occurs. Adding the locality restriction, this use of data flow models is perfectly compatible with object-oriented modelling. A consequence of the locality restriction is that when an action in an OOA state model has side-effects upon other objects, then this action should be split into several cooperating local actions. We return to this point when we analyse object communication below. Note that the locality restriction takes away the need to represent synchronous communication during an action in an OAM.

OOA also allows the guard of a transition in a state model to be global, that is, it may test the state of any object in the system. This does not involve side-effects on other objects. The effect of evaluating the guard is purely local, namely, enabling or disabling an object transaction. We therefore follow OOA in not imposing a locality restriction on guards.

\subsection{Formalisation}

To illustrate the formalisation of the process model, Fig. 12 contains a specification of the ACCOUNT object class. This specification assumes that there is a CUSTOMER object class with key attribute customer_ID that has an inverse function inv_customer_ID. It also assumes that a value type $A C C O U N T \_S T A T E$ has been specified containing only two values, OVERDRAWN and IN_GOOD_STANDING. All possible actions of $A C C O U N T$ objects are declared in the transitions section of the specification, shown in Fig. 13.

Figure 13 shows a declarative formalisation of the guards and transactions of the ACCOUNT object class. The axioms use dynamic logic to define guards and effects of transitions. There are two kinds of axioms; state axioms and transition axioms.

- A state axiom is a sentence, not containing modal operators, that must be true in all states of the system. There is a state axiom in the account specification that says that the customer of an existing account must exist. Note that the customer $I D$ attribute of CUSTOMER is overloaded. 
- There are two kinds of transition axioms, namely, guarding and effect axioms.

- A guarding axiom has the form $\langle\alpha>$ true $\rightarrow \emptyset$. This means that if the transition $\alpha$ leads to a next state, then (currently), $\varnothing$ is true. In other words, if $\varnothing$ is false, then $\alpha$ does not lead to a next state. We call the conjunction of all consequents of guarding axioms for $\alpha$ the guard of $\alpha$. Truth of the guard is a necessary condition for $\alpha$ to occur.

- Effect axioms have the form $\emptyset \rightarrow[\alpha] \psi$. This means that if the precondition $\emptyset$ is currently true, then after execution of $\alpha$ the postcondition $\psi$ is true.

Recall that in subsection 4.3, we defined the intended semantics as a state transition system in which all states share the abstract data types, including the identifier types. The states only differ in the interpretation that they give to the attribute and local predicate names of objects. This includes the Exists and Used predicates, which may have different extensions in different possible states. In this structure, the state axioms act as static integrity constraints that restrict the set of possible states of the system.

The transition names declared in a class are interpreted as possible state transitions for the class instances. The transition axioms restrict the possible transitions that an instance can take. These axioms do not uniquely determine the possible transitions. It is for example compatible with the axioms that increase balance changes the customer_ID attribute of the account. It is also compatible with the axioms that if the guard of a transition is true, the transition cannot be taken: what is specified is merely that if the guard is false, the transition cannot be taken. To make the intended meaning explicit, we should state that the intended transition relation must satisfy the frame assumption and the qualification assumption. We state these assumptions informally:

- The frame assumption says that a transition relation brings about is the smallest change compatible with the effect axioms.

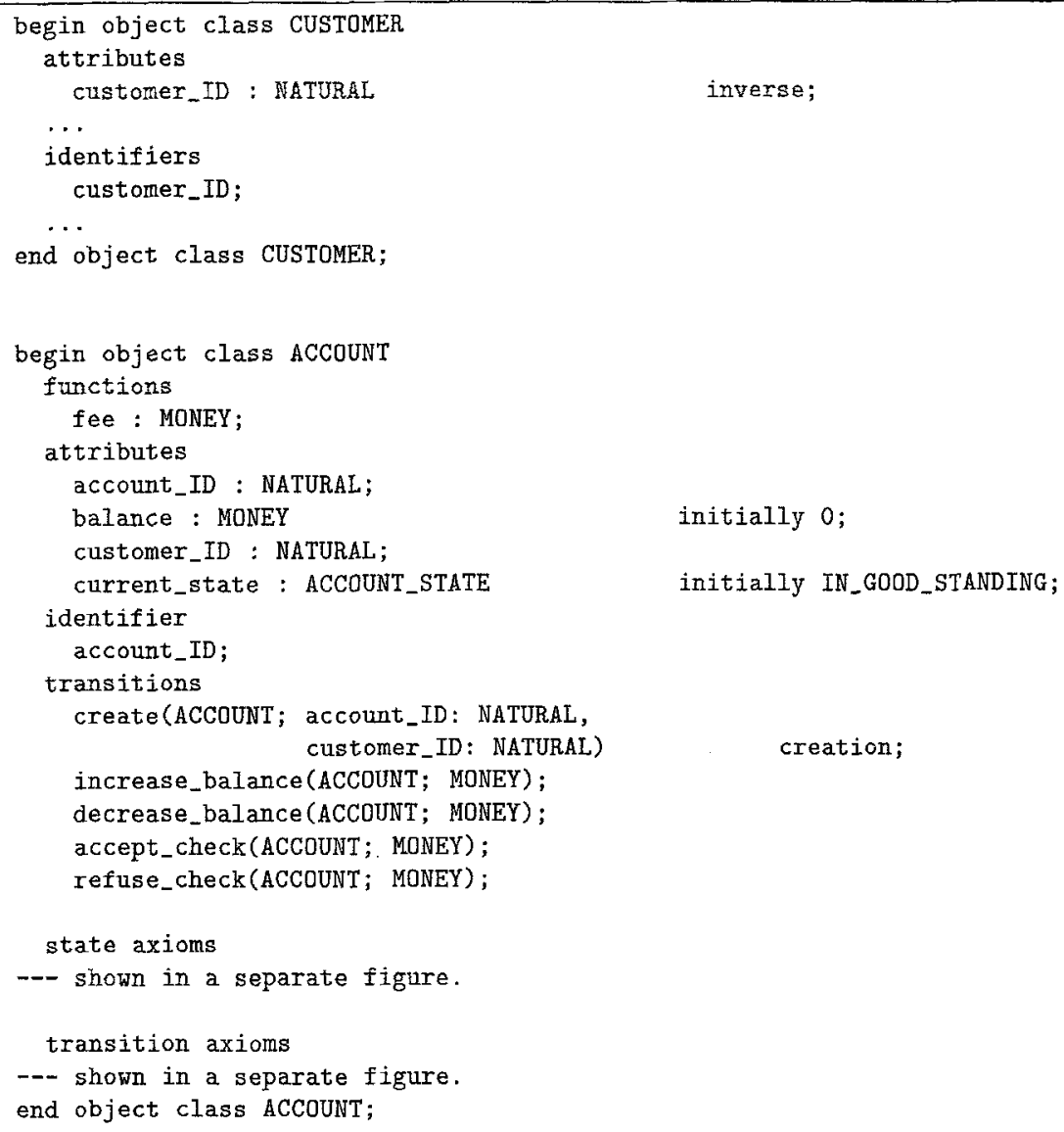

Fig. 12. The CUSTOMER class has customer_ID attributes, that is unique on existing customers and that has an inverse attribute. 
- The qualification assumption says that any transition compatible with the guard axioms can be taken.

We can summarise this by the slogan that we have a minimal change/maximal reachability semantics. It is well-known that there are different possible formalisations of minimal change and we indicate below which choices have been made in LCM. The assumptions are not expressible as first-order axioms. One way around this is to generate frame and guard sufficiency axioms for each individual specification automatically [32]. Another way of dealing with this is to translate the dynamic logic into first-order calculus, making the assumptions explicit in the translation process [49]. In both approaches, the following problems have to be dealt with.
- Due to the locality assumption, we know that effect axioms have only one identifier variable, which denotes the object executing the transition. However, if we would add the postcondition that for all other objects, nothing changes, then synchronous execution of severaltransition would become impossible. Each transition would prohibit any synchronously executing transition from performing any change on the object that executes it. The solution to this is to apply the locality assumption only to system transactions: a system transaction changes only the state of the objects that participate in it and leaves the state of all other objects invariant. For any particular transaction, this can be expressed as first-order axioms. We return to this when we discuss system transactions.

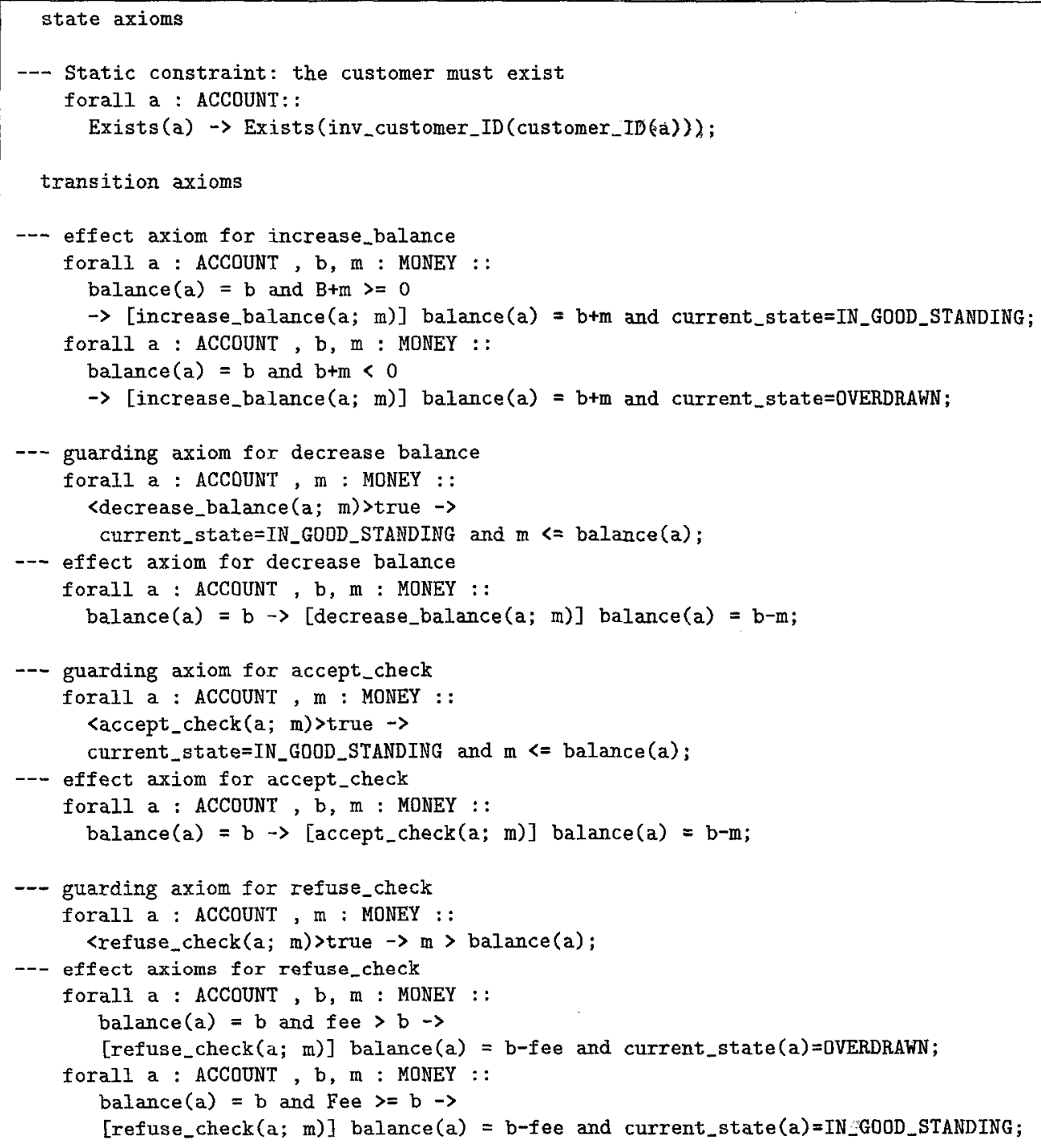

Fig. 13. Transition axioms for the ACCOUNT object class. 
- Within a single object, a transition axiom may change several local attributes and predicates. The simplest assumption here is to assume that any attribute and predicate that does not occur in the postcondition remains unchanged. For example, we can add the axiom that increase_balance does not change the attribute customer_ID. The problem with this approach is that some transitions may imply derived updates of the object state. We haveshiown elsewhere how to deal with this when derivation rules have the form of Horn clauses [50]. We are currently looking at ways to generalise this approach.

- The qualification assumption can be formalised in a simple way by conjoining all guards of a transition and declaring these necessary as well as sufficient for a transition to be possible. This is a kind of "guard completion". The problem with this is that in a specialisation of the class, additional guards may be specified for the transition. The solution to this is to apply guard completion only to the most specific classes, where all constraints on a transition are known.

Returning to the formalisation of the OOA techniques, we remark that the state model of a class is formalised simply by introducing a state variable and updating this variable in the effect axioms. The actions performed by a state model appear as transitions in the LCM specification and the effect axioms define the effect of these actions on the local state of the object. The effect axioms thus provide the essential information about object transactions as represented in the essential data flow model of these transactions. Finally, the guard axioms formalise the guards that we. added to the statemodels in our methodological analysis of Section 4. The informal specifications of object transactions such as shown in Fig. 11 are really paraphrases of the effect and guard axioms. Note that in the formalisation we do not distinguish input from output parameters of actions; this is extra information provided by the essential data flow diagram that may be useful to the programmer who implements the transaction. This leaves the communication structure of the model to be formalised, as represented by the triggering mechanism of the state models. We turn to this in the next section.

\section{The Object Communication Model}

\subsection{Structure}

The object communication model (OCM) showssmessage sending between objects. The OCM is represented as a directed graph in which the nodes are external entities or individual objects and the arrows are actions sent by one node to another. The graph can be constructed from the state models: each action generated by a state model is sent to some state model or to an external entity. For the sender, the message is a generated action; for the receiver, it is an event to which it may have to respond. Message-sending in OOA is asynchronous because the sender can continue its activity immediately after it has sent a message. A message generated by one object is received by the receiving object at the instant in which they are generated. Messages never get lost [17, p. 107].

The actions that an object performs are atomic in the sense that they cannot be broken down in subactions but they are assumed to take time [17, pp. 45 and 105]. If a receiver is busy when it receives a message, it will be processed later. OOA assumes partial arrival order non-determinism: messages generated by the same object will be processed in the order in which they are sent, but messages sent by different objects may be processed in any order.

\subsection{Methodological Analysis}

OOA implicitly assumes the fairness assumption that an object will not wait to process an event for an indefinitely long time. When an object is ready to process an event, then it will not delay processing this event further. This assumption is not mentioned by Shlaer and Mellor.

The assumption of partial arrival order non-determinism causes problems: first, if two timers send a timeout to an object simultaneously, one of these must wait, which defeats the purpose of the timeout. Timeouts have the essential and annoying property that you cannot postpone them. Second, if two timers send timeouts to the same object at different moments, then these may be processed in a reverse order!

These problems disappear once we assume that actions do not take time. This is known as the synchrony hypothesis in real-time specification theory $[19,51,52]$. It ties in well with the assumption of perfect technology mentioned earlier, known from classical structured analysis [41]. In a requirements specification, we avoid any implementation bias by pretending to specify the observable behaviour of a system with infinite processing speed. Our account of object transactions implies that objects too satisfy the synchrony hypothesis: in an object transaction, the action occurs simultaneously with the event that triggers it. The synchrony hypothesis considerably simplifies the requirements specification. At the same time, it is less unrealistic as it seems, 
because it is a requirement on the implementation of the system that it responds sufficiently fast to external stimuli so that the stimulus-response time can be considered to be zero from the standpoint of the environment. If an activity of the system takes a nonnegligible amount of time then we should specify this in the requirements as a sequence of two instantaneous actions, which represent the start and the end of this activity.

As a further simplification we replace the model of asynchronous message-sending with the model of synchronous message-sending. In this model, the sender must wait until the receiver is ready to receive the message. This does not cause a loss of generality, for

\begin{tabular}{|l|l|l|l|l|}
\hline ACCDUNT & open_account & deposit & withdray & submit_check \\
\hline create & $\begin{array}{l}\text { increase_ } \\
\text { balance }\end{array}$ & $\begin{array}{l}\text { decrease_ } \\
\text { balance }\end{array}$ & $\begin{array}{l}\text { accept_check } \\
\text { refuse_check }\end{array}$ \\
\hline
\end{tabular}

Fig. 14. Transaction decomposition table of ACCOUNT transactions.

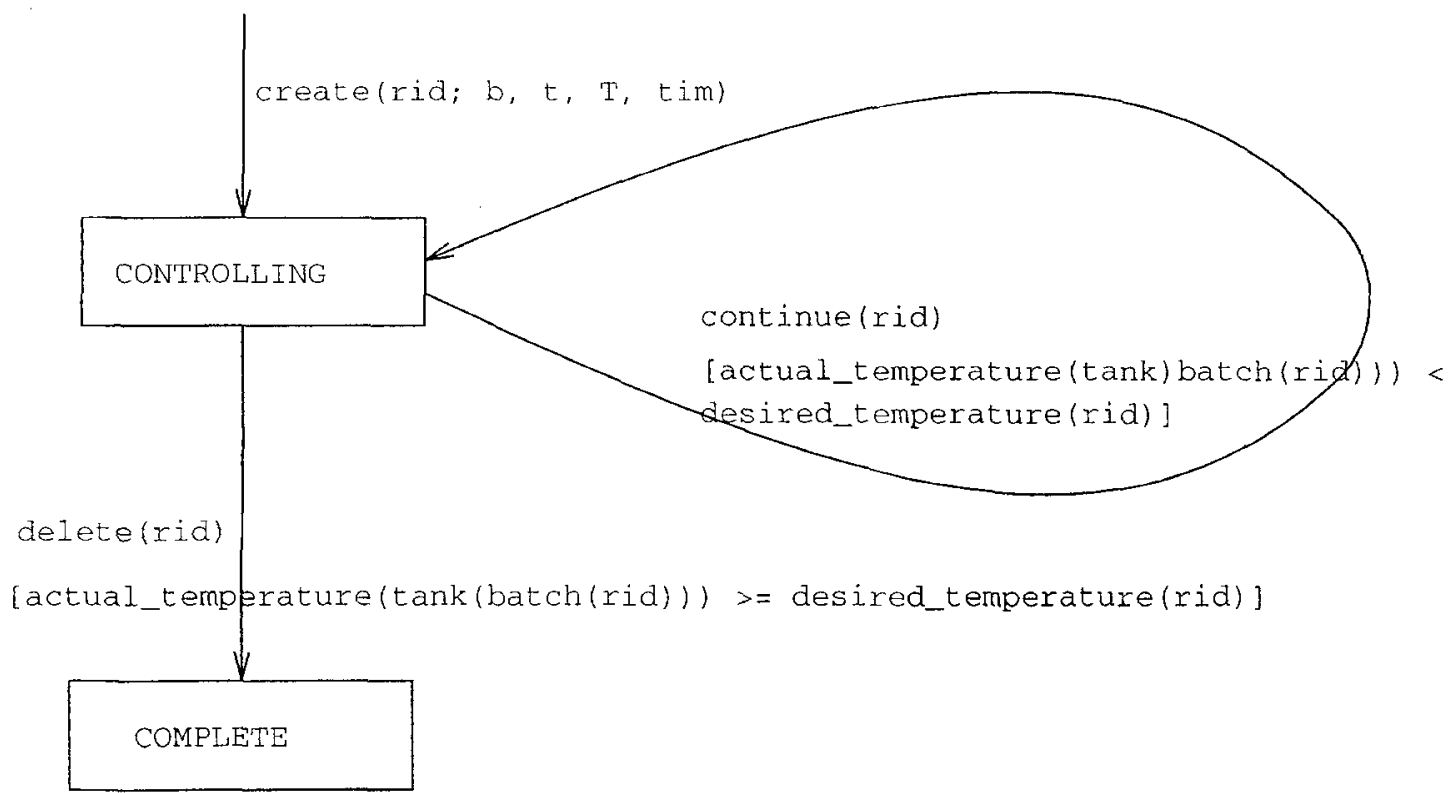

Fig. 15. Simplified life cycle of the temperature ramp.

\begin{tabular}{|c|c|c|c|}
\hline & $\begin{array}{l}\text { start_controlling- } \\
\text { temperature }\end{array}$ & continue_heating & stopheating \\
\hline BATCH & do_temperature_ramp & & $\begin{array}{l}\text { temperature_ramp_ } \\
\text { complete }\end{array}$ \\
\hline $\begin{array}{l}\text { TEMPERATURE_- } \\
\text { RAMP }\end{array}$ & create & continue & delete \\
\hline TIMER & create_timer & $\begin{array}{l}\text { timer_expires and } \\
\text { set_timer }\end{array}$ & $\begin{array}{l}\text { timer_expires and } \\
\text { delete_timer }\end{array}$ \\
\hline HEATER & turn_on & & \\
\hline
\end{tabular}

Fig. 16. Transaction decomposition table of some juice controller transactions. 
asynchronous communication can be modelled by introducing communication channels as objects that communicate synchronously with senders and receivers. The behaviour of these channel objects must be explicitly specified and this makes explicit whatever time loss may occur between the beginning and the end of an asynchronous communication chain.

Adding the synchronicity assumption, the model of synchronous communication, and the earlier restriction of local action effects, we now have the following model of system transactions: Each system transaction is an atomic occurrence that consists of a stimulus from the environment of the system that triggers a number of local object transactions, which may result in a change of state of the (objects in the) system and in the sending of a response to the environment of the system; all of this occurs in one instant of time. We can represent this in tabular form by a transaction decomposition table as shown in Fig. 14. The top row of the table lists system transactions, the leftmost column lists classes and the entries list the action by which a class instance participates in the transaction. The system transactions are the interactions between the system and its environment. Figure 14 shows a transaction decomposition table for the bank account system. The submit_check event may trigger an accept_check or refuse_check response, depending on the evaluation of the guards of these transitions. This transaction decomposition table is extremely simple because each system transaction is decomposed into exactly one object transaction.

The transaction decomposition table of the juice plant controller is more interesting. Figure 15 shows the result of transforming the Moore into a Mealy representation, removing transitory states and adding declarative guard specifications. To simplify the representation further, we also assumed that the precondition for creating the ramp is tested before creation. This is different from Fig. 9, where this precondition is tested when the state model arrives in the transitory Created state. Figure 16 gives the transaction decomposition table of some of the juice plant controller transactions. Note that the continue heating transaction involves two actions in a TIMER object: timer_expires and set_timer. Since these actions occur (conceptually) at the same time, atomicity of the transaction is not violated. The transaction decomposition table does not represent the initiative of a transaction; it merely represents which local actions participate in a transaction without distinguishing cause and effect.

The transaction decomposition table is an example of a well-known device in structured analysis to correlate data structures with system functions. In Information Engineering one correlates system functions with entity types in a similar matrix $[53,54]$ and the Shumate-
Keller method of structured analysis for real-time systems offers a similar technique, called the action/ data store table [9], to find a data flow model of a system. Although the table is not used in structured analysis to represent object communication within a system transaction, the differences are smaller than the similarity. It is therefore a central tool in a toolkit for structured as well as object-oriented modelling.

The information in a transaction decomposition table can be supplemented with an essential data flow diagram (DFD) of the transaction. Figure 17 shows such a DFD for the transaction start_controlling _ temperature of the juice plant controller. Because the actions participating in a transaction have local effects, a specification of a transaction can be obtained by conjoining the specifications of these local effects. For example, the specification of the effect of the start controlling temperature transaction is given in Fig. 18.

Turning now to the OCM of OOA, we notice that this summarises information about message sending between objects that can be constructed from the state models of the object classes. The communications that occur during a system transaction are now represented in the transaction decomposition table, but we already mentioned that cause-effect relations are not represented in the table. We can represent these relations by constructing an OCM. The OCM for the start_controlling_temperature transaction constructed from the Mealy model of Fig. 15 is shown in Fig. 19. This differs from the OCM that would be derived from the Moore model of Fig. 9 because we assume that the precondition for creating the temperature ramp is tested before the ramp is created.

Note that the transaction decomposition table or the OCM cannot represent arbitrary causal chains of undetermined length. In the Statemate approach, a stimulus to the system can trigger cause-effect chains whose length may depend upon the stimulus parameters and the current state of the system $[19,55]$. These chains cannot be represented by an OCM or a transaction decomposition table, because they cannot be determined in advance of execution time.

\subsection{Formalisation}

Our methodological analysis has led to a system transaction concept that is simple to formalise: each system transaction is a synchronous execution of a nonempty finite set of local object transactions. Because each object transaction has a local effect only, this composition is harmless and we can simply conjoin the local effects. This is the formal reason why requiring transitions to have local effects only is advantageous. 


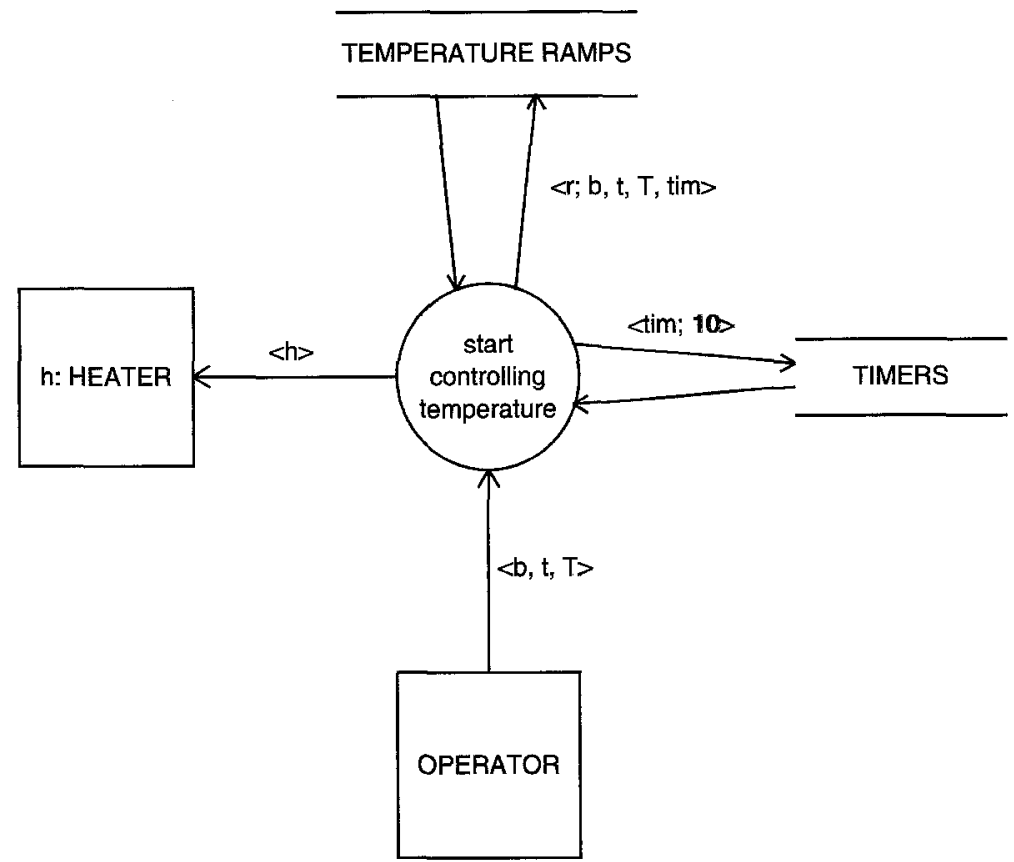

Fig. 17. Data flow diagram of the start_controlling_temperature transaction of the juice plant controller.

Figure 20 shows the transaction specifications of the juice plant control system corresponding to the transaction decomposition table in Fig. 16. Each transaction is a synchronous composition (represented by the symbol \&) of local transitions from different objects. The semantics of this operator is easily accommodated by the semantics expounded so far. Recall that the different states of the system share the same collection of abstract data types and that the only difference between the states is the interpretation of the local attributes and predicates of the objects. States are linked by state transitions. We now require state transitions to be labelled by non-empty finite sets of object transitions. If $\alpha_{1}$ and $\alpha_{2}$ are actions such that $\alpha_{1}$; $\alpha_{2}$ has the same effect as $\alpha_{2} ; \alpha_{1}$, then we define the effect of $\alpha_{1} \& \alpha_{2}$ to be the effect of an (arbitrary) sequential execution of $\alpha_{1}$ and $\alpha_{2}$. If the effect of $\alpha_{1} ; \alpha_{2}$ is different from the effect of $\alpha_{2} ; \alpha_{1}$, then the effect of $\alpha_{1} \& \alpha_{2}$ is undefined. In that case, $\alpha_{1} \& \alpha_{2}$ does not lead to a next possible state of the system. The dynamic logic inference axiom that can be used to reason about synchronous execution is

$$
\left[\alpha_{1}\right] \emptyset_{1} \wedge\left[\alpha_{2}\right] \emptyset_{2} \rightarrow\left[\alpha_{1} \& \alpha_{2}\right] \emptyset_{1} \wedge \emptyset_{2}
$$

This axiom was first given by Meyer [56] and is assumed in LCM [34]. A detailed analysis of possible interpretations of \& is given by Wieringa and Meyer [57].

Note that we allow local synchronicity by allowing an object to participate in a system transaction by several local actions. For example, in Fig. 16, a TIMER

start_controlling-temperature(b: BATCH, end_time: TIME, desired_temperature:

RATIONAL, $r$ : TEMPERATURE_RAMP, $h$ : HEATING_TANK, tim : TIMER)

- do_temperature.ramp $(b ; t, T)$ is a transition of batch $b$ to create a temperature ramp with end time end_time and desired temperature desired_temperature.

- $\operatorname{create}(r ; b, t, T, t i m)$ creates a fresh temperature ramp $r$, sets the start_time of $r$ to the current time of the clock, sets the start_temperature to the current temperature of the heating tank assigned to the batch $\mathrm{b}$, sets the end time to end_time and the desired temperature to desired-temperature. The timer identifier tim is stored in attribute tt timer and the temperature ramp goes into state CONTROLLING.

- create_timer(tim, 10) creates a timer with a fresh identifier tim and sets the deadline to 10 time units.

- turn_on_heater(h) turns heater $h$ on.

Fig. 18. Construction of a specification of the start_controlling-temperature transaction by conjunction of the local transitionspecifications. 


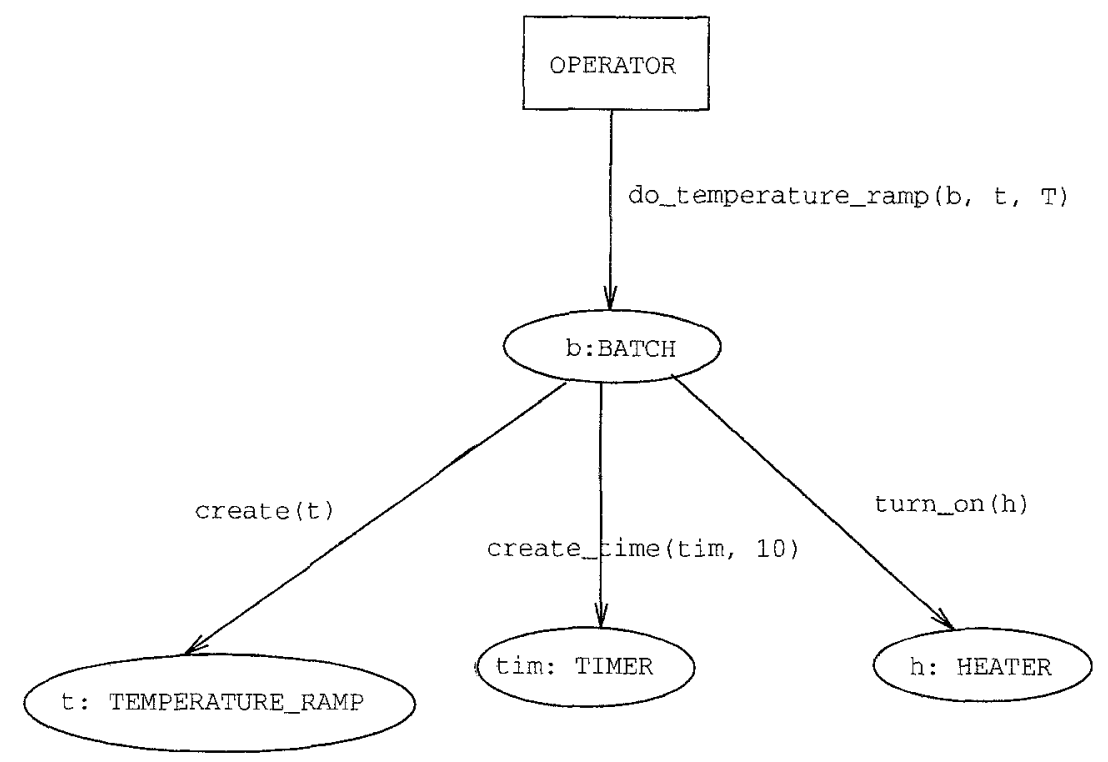

Fig. 19. OCM of the transaction start_controlling_temperature $(b, t, T, t, h$, tim) constructed from the Mealy model.

participates in the continue_heating transaction by the actions timer_expires and set_timer. This is meaningful only if these local actions do not interfere, that is, the effect of all possible sequential executions must be the same. This is the case in our example. In general, the requirement of non-interference of synchronously executed local actions must be verified by the specifier. In what follows, an object transaction is the finite set of one or more transitions by which an object participates in a system transaction.

We now have sufficient ammunition to give a semantics to system transactions. We know that a system transaction is a finite set of object transactions $\alpha_{1}, \ldots, \alpha_{n}(n \geq 1)$ executed by different objects, and that each object transaction has a local effect. We therefore know that all sequential executions of these local actions have the same cumulative effect, so that their effects can be simply conjoined. More precisely, we have the following:

- The effect of the system transaction $t \alpha_{1} \& \ldots \alpha_{n}$ is given by the effect axiom $\Phi \rightarrow[t] \psi$, where $\Phi$ is the conjunction of all preconditions and $\psi$ the conjunction of all postconditions of $\alpha_{\dot{\boldsymbol{j}}} i=1, \ldots n$. The locality assumption is applied to this transaction effect axiom by stating that all objects not participating in the transaction, and all attributes and predicates not mentioned in the transaction effect axiom, do not change.

- The guard $g$ of a system transaction $t$ is the conjunction of the guards of the participating local object transactions. This guard is defined to be necessary and sufficient for the possibility of $<t>$ true $\leftrightarrow g$.

This means that we check all guards of a system transaction before the transaction is performed and can then perform the object transactions that participate in the system transactions in any arbitrary order as one atomic occurrence.

\section{Discussion and Conclusions}

In the introduction we claimed that the definition of conceptual tools for requirements engineering should be done by formal means even if the tools themselves are semi-formal. In this paper, we have substantiated this claim by analysing the semi-formal techniques in OOA by formal means. This resulted in a simplification of the semi-formal tools so as to realise a closer match between the semi-formal and formal specification of system requirements. In many requirements specification cases, a formal specification of requirements may never be produced but it should be clear that the benefits of formalisability extend to all cases where the semi-formal techniques are used. We proposed the following simplifications of the techniques in OOA.

- Formalisation allows us to pinpoint the difference between objects and links as the presence of a single or a tuple of identifiers. In all other aspects, objects and links can be treated in the same way. This allowed us to propose a simplification of the diagram technique for "associative objects". 
- A formal analysis also uncovered an essential difference between keys (unique across an existence set) and identifiers (unique across all possible objects) and introduce semi-formal notations accordingly.

- The state model was simplified by using results from automata theory about the equivalence of Moore and Mealy automata and from formal specification theory about declarative guard specification.

- The process model was simplified considerably by specifying the effects of object transactions formally and adjusting the data flow technique and corresponding informal process specification accordingly. In order to simplify formalisation, we adopted the object-oriented principle of localisation of action effects and borrowed the synchronicity assumption from formal specification theory; this ties in well with the essential modelling philosophy of structured analysis.

- The communication model was simplified by requiring all communications to be synchronous. This simplifies formalisation of system transactions as finite sets of synchronously executing local object transactions. The resulting semi-formal technique is conceptual simpler than the asynchronous communication model of OOA.

The result is a collection of semi-formal tools that differs from OOA. One may wonder why we do not formalize OOA techniques just as they are. The answer is that this would have made the formal counterpart of the semi-formal techniques unnecessarily complex. The

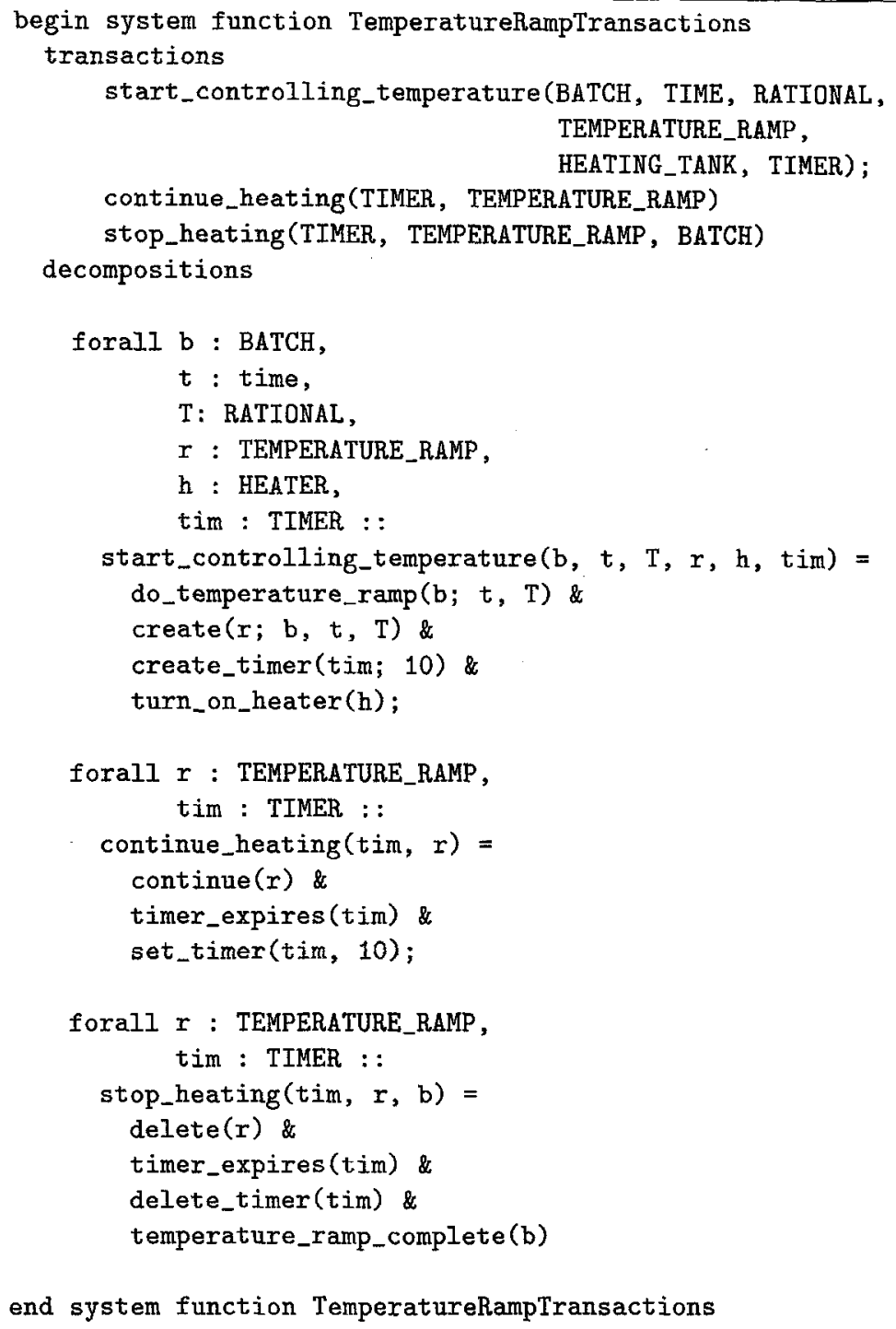

Fig. 20. Specification of two transactions of the juice controller. 
simplifications brought about by a precise analysis of object identification, behaviour and communication are motivated by the desire to formalise these aspects but as a side-effect they make a simpler semi-formal presentation of these aspects possible. We should benefit from this by applying these simplifications to the semi-formal techniques. In general, we should not expect formalisation of semi-formal techniques without modification of those techniques.

The modified techniques have a clearer semantics and, correspondingly, clearer directions for use than the techniques as defined in OOA. This should lead to improved quality of the specifications delivered in requirements engineering.

We believe that formalisation not only simplifies the semi-formal techniques, it also enhances our understanding of the meaning and use of those techniques. Our analysis of OOA and of other semi-formal methods leads to the identification of a common framework for structured and object-oriented requirements specification techniques that can be used to define a toolkit of semi-formal and formal techniques. We cannot go into detail about this framework here but the contours of this kernel are that a specification of software product requirements always consists of an

- object model,

- a model of object communications, and

- a model of the way these communications are used in system transactions.

Structured and object-oriented methods agree on this framework but differ in the way they populate it with techniques and heuristics. In particular, the heuristics for modularising the system differ. A toolkit of techniques for requirements specification should be based upon this framework. We believe that the simplified techniques as defined in this paper have a place in a toolkit. Detailed analysis of the framework and the tools to be put in the toolkit is part of current research. Here, we must emphasise that a toolkit approach to requirements specification does not contradict a methodical way of working. Rather, tools can be defined with a precise semantics and corresponding directions for use, in such a way that they can be used in different methods. This is not different from the fact that the same project management techniques can be used in different kinds of project organisations, with different development strategies, etc.

Turning to the formal counterpart of the semi-formal techniques, we note that the transaction decomposition table plays a central role in this. A formal language that can act as counterpart of the semi-formal techniques described in this paper should be able to specify object classes with attributes, local actions and action guards, and system transactions that are realised by a finite set of communicating objects as represented by the transaction decomposition table. LCM was designed with this purpose in mind but other formal languages can also do the job, such as Troll $[25,58]$ or Oblog [59]. One of the benefits of combining formal specification techniques with semi-formal techniques is the increased usability of the formal techniques. Because semi-formal techniques are actually used on a larger scale than formal techniques, another benefit is that the attempt to define such a combination leads to the identification of useful constructs to include in the formal language.

Our current research focuses on the definition of what we may call a standard model of requirements specifications, which consists of a framework that can accomodate structured as well as object-oriented techniques and that is populated with structured as well as object-oriented techniques. We are currently applying this framework to several realistic case studies. On the formal side, we are studying techniques for reachability analysis of systems specified in LCM. The result of a reachability analysis may be a possible system scenario, which can then be used to validate the requirements as well as to define acceptance tests. We are also looking at ways to make the transaction specification mechanism of LCM more powerful, so that a transaction may involve a finite but indeterminate set of objects that cooperate to achieve the transaction result.

\section{Acknowledgements}

The authors wish to thank the anonymous referees for their detailed and stimulating comments on an earlier version of the paper. These comments forced us to rethink the arguments in the paper and led to improvements in the presentation. The work was partially supported by ESPRIT BRA WG 6071 IS-CORE and by ESPRIT BRA WG 8319 Model Age.

\section{References}

1. Jones, J. C. Design methods: seeds of human futures. Wiley, New York, 1970

2. Jensen, R. W. and Tonies, C. C. Software engineering. Prentice-Hall, Englewood Cliffs, NJ, 1979

3. Birrell, N. D. and Ould, M. A. Practical handbook for software development. Cambridge University Press, Cambridge, 1985

4. Wieringa, R. J. Requirements engineering: frameworks for understanding. Wiley, New York, 1996.

5. Wieringa, R. J. A method for building and evaluating formal specifications of object-oriented conceptual models of database systems (MCM). Technical Report IR340, Faculty of Mathematics and Computer Science, Vrije Universiteit, December 1993 
6. Gomaa, H. Software design methods for concurrent and real-time systems. Addison-Wesley, Reading, MA, 1993

7. Hatley, D. and Pirbhai, I. Strategies for real-time system specification. Dorset House, 1987

8. i Logix. The Languages of STATEMATE. Technical report, i-Logix Inc., 22 Third Avenue, Burlington, MA. 01803, USA, January 1991

9. Shumate, K. and Keller, M. Software specification and design: A disciplined approach for real-time systems. Wiley, New York, 1992

10. Ward, P. T. and Mellor, S. J. Structured development for real-time systems. Prentice-Hall/Yourdon Press, 1985. Three volumes

11. Booch, G. Object-oriented design with applications, 2nd edn. Benjamin/Cummings, 1994

12. Coad, P, and Yourdon, E. Object-oriented analysis. Yourdon Press/Prentice-Hall, 1990

13. Embley, D. W., Kurtz, B. D. and Woodfield, S. N. Objectoriented systems analysis: A model-driven approach. Prentice-Hall, Englewood Cliffs, NJ, 1992.

14. Jacobson, I. Christerson, M., Johnsson, P. and Övergaard, G. Object-oriented software engineering: A use case driven approach. Prentice-Hall, Englewood Cliff, NJ, 1992

15. Martin, J. and Odell, J. Object-oriented methods: A foundation. Prentice-Hall, Englewood Cliffs, NJ, 1995.

16. Shlaer, S. and Mellor, S. J. Object-oriented systems analysis: modeling the world in data. Prentice-Hall, Englewood Cliffs, NJ, 1988

17. Shlaer, S. and Mellor, S. J. Object lifecycles: modeling the world in states. Prentice-Hall, Englewood Cliffs, NJ, 1992

18. Rumbaugh, J., Blaha, M., Premerlani, W., Eddy, F., and Lorensen, W. Object-oriented modeling and design. Prentice-Hall, Englewood Cliffs, NJ, 1991

19. i Logix. The Semantics of Statecharts. Technical report, i-Logix Inc., 22 Third Avenue, Burlington, MA. 01803, USA, January 1991

20. i-Logix Inc., 22 Third Avenue, Burlington, MA. 01803, USA. STATEMATE Analyzer Reference Manual Version 5.0, 1993

21. France, R. B. and Larrondo-Petrie, M. M. From structured analysis to formal specification: state of the theory. In Proceedings of the 1994 ACM Computer Science Conference, 1994

22. Bates, B. W., Bruel, J. -M., France, R. B. and LarrondePetrie, M. M. Guidelines for the formalizing Fusion object-oriented analysis models. In P. Constantopoulos, J. Mylopoulos, and Y. Vassiliou, eds., Advanced Information Systems Engineering (CAiSE'96), pp. 222-233. Springer, 1996. Lecture Notes in Computer Science 1080

23. Feijs, L. M. G., Jonkers, H. B. M. and Middelburg, C. A. Notations for Software Design. Springer, Berlin, 1994

24. Bourdeau, R. H. and Cheng, B. H. C. A formal semantics for object model diagrams. IEEE Transactions on Software Engineering, 21(10): 799-821, October 1995

25. Jungclaus, R., Saake, G., Hartmann, T. and Sernadas, C. Troll: A language for object-oriented specification of information systems. ACM Transactions on Information Systems, 14(2): 175-211, April 1996

26. Wieringa, R. J. Combining static and dynamic modeling methods: A comparison of four methods. Computer Journal, 38(1): 17-30, 1995
27. Chen, P. P. -S. The entity-relationship model: Toward a unified view of data. ACM Transactions on Database Systems, 1: 9-36, 1976

28. Smith, J. M. and Smith, D. C. P. Database abstractions: Aggregation and generalisation. ACM Transactions on Database Systems, 2: 105-133, 1977

29. Smith, J. M. and Smith, D. C. P. Database abstractions: Aggregation. Communications of the ACM, 20: 405-413, June 1977

30. Kent, W. A rigorous model of object reference, identity, and existence. Journal of Object-Oriented Programming, 4(3): 28-36, June 1991

31. Wieringa, R. J. and de Jonge, W. Object identifiers, keys, and surrogates. Theory and Practice of Object Systems, 1(2): 101-114, 1995

32. Wieringa, R. J., de Jonge, W., and Spruit, P. A. Using dynamic classes and role classes to model object migration. Theory and Practice of Object Systems, 1(1): $61-83,1995$

33. Wieringa, R. J. Equational specification of dynamic objects. In Meersman, R. A., Kent, W., and Khosla, S. eds., Object-oriented databases: analysis, design, and construction (DS-4), pp. 415-438. North-Holland, Amsterdam, 1991

34. Wieringa, R. J. A formalisation of objects using equational dynamic logic. In Delobel, C., Kifer, M., and Masunaga, Y. eds., 2nd International Conference on Deductive and Object-Oriented Databases (DOOD'91), pp. 431-452. Springer, Berlin, 1991. Lecture Notes in Computer Science 566

35. Ehrich, H. -D., Gogolla, M. and Lipeck, U. W. Algebraische Spezifikation abstrakter Datentypen. Teubner, B. G., 1989

36. Ehrig, H., Mahr, B. and Orejas, F. Introduction to algebraic specification. Part 1: Formal methods for software development. Computer Journal, 35(5): $460-477,1992$

37. Goguen, J. A. and Meseguer, J. Order-sorted algebra I: Equational deduction for multiple inheritance, overloading, exceptions and partial operations. Theoretical Computer Science, 105: 217-273, 1992

39. Hopcroft, J. E. and Ullman, J. D. Introduction to Automata Theory, Languages and Computation. Addison-Wesley, Reading, MA, 1979

40. Gray, J. The transaction concept: virtues and limitations. In Zaniolo, C. and Delobel, C. eds., Proceedings of the Seventh International Conference on Very Large Databases, pp. 144-154, Cannes, France, September 9-11 1981

41. McMenamin, S. M. and Palmer, J. F. Essential Systems Analysis. Yourdon Press/Prentice Hall, 1984

42. Harel, D. Statecharts: a visual formalism for complex systems. Science of Computer Programming, 8: 231-274, 1987

43. Jungclaus, R., Wieringa, R. J., Hartel, P., Saake, G. and Hartmann, T. Combining Troll with the Object Modeling Technique. In Wolfinger, B. ed., Innovationen bei Rechen- und Kommunikationssystemen. GI-Fachgespräch FG 1: Integration von semi-formalen und formalen Methoden für die Spezifikation von Software, pp. 35-42. Springer, Informatik aktuell, Berlin, 1994

44. Shlaer, S. and Mellor, S. J. An object-oriented approach to domain analysis. ACM SIGSOFT Software Engineering Notes, 14(5): 66-77, July 1989 
45. Wieringa, R. J., Jungclaus, R., Hartel, P., Saake, G. and Hartmann, T. OMTROLL - Object modeling in troll. Proceedings of the international workshop on information systems -- correctness and reusability (ISCORE'93), Lipeck Udo W. and Koschorrek, G. (eds.), pp. 267-283. Institut für Informatik, Universität Hannover, Postfach 6009, D-30060, Hannover., September 1993

46. Booch, G. Object-oriented development. IEEE Transactions on Software Engineering, SE-12: 211-221, 1986

47. Hayes, F. and Coleman, D. Coherent models for objectoriented analysis. In Paepcke, A., ed., Object-Oriented Programming: Systems, Languages and Applications/ European Conference on Object-Oriented Programming, pp. 171-183. ACM, 1991. Sigplan Notices 25, no 11

48. Rumbaugh, J. OMT: The functional model. Journal of Object-Oriented Programming, 8(1): 10-14, MarchApril 1995

49. Feenstra, R. B. and Wieringa, R. J. Translating LCM specifications into reachability calculus. In preparation

50. Spruit, P. A., Wieringa, R. J., and Ch. Meyer, J. -J. Axiomatisation, declarative semantics and operational semantics of passive and active updates in logic databases. Journal of Logic and Computation, 5(1): 27-50, 1995

51. Berry, G. and Cosserat, I. The ESTEREL synchronous programming language and its mathematical semantics.
In Brookes, S. and Winskel, G. eds., Seminar on Concurrency, pp. 389-448, 1985. Lecture Notes in Computer Science 197

52. Harel, D. and Pnueli, A. On the development of reactive systems. In Apt, K. ed., Logics and models of concurrent systems, pp. 477-498. Springer, 1985. NATO ASI Series

53. Martin, J. Strategic data-planning methodologies. Prentice-Hall, Englewood Cliffs, NJ, 1982

54. Martin, J. Information Engineering, Book II: Planning and analysis. Prentice-Hall, Englewood Cliffs, NJ, 1989

55. Harel, D., Pnueli, A., Schmidt, J. P. and Sherman, R. On the formal semantics of statecharts. In proceedings, symposium on logic in computer Science, pp. 54-64. Computer Science Press, June 22-25 1987

56. Meyer, J. -J. Ch. A different approach to deontic logic: Deontic logic viewed as a variant of dynamic logic. Notre Dame Journal of Formal Logic, 29: 109-136, 1988

57. Wieringa, R. J. and Meyer, J. -J. Ch. Actors, actions, and initiative in normative system specification. Annals of mathematics and artificial intelligence, 7: 289-346, 1993.

58. Hartmann, T., Saake, G., Hartel, P. and Kusch. J. Revised version of the modelling language TROLL (TROLL version 2. 0). Technical Report 94-03, Abt. Datenbanken, Tech. Universität Braunschweig, P. B. 3329, Braunschweig, Germany, April 1994

59. Oblog Software. OBLOG Case V1. 2, 1995 